Chinese Journal of Organic Chemistry

\title{
氧头孢衍生物不对称合成的研究进展
}

\author{
付冬君 ${ }^{a}$ 刘英超 ${ }^{a}$ 李 锋 ${ }^{a}$ 张 恩 ${ }^{a}$ 刘宏民 ${ }^{*, a, b}$ \\ ( ${ }^{a}$ 郑州大学药学院 郑州 450001) \\ $\left({ }^{b}\right.$ 郑州大学新药研发中心 郑州 450001)
}

\begin{abstract}
摘要 氧头孢是 $\beta$-内酰胺类药物的代表性母核, 其衍生物显著的生物学活性和合成的复杂性对药物化学家极具吸引力. 以氧头孢合成中使用的关键方法分类, 对氧头孢的合成研究进展进行了综述.

关键词 氧头孢烯; 氧头孢烷; 不对称合成; 抗生素
\end{abstract}

\section{Recent Advances in Asymmetric Synthesis of Oxacephems}

\author{
Fu, Dongjun ${ }^{a} \quad$ Liu, Yingchao ${ }^{a} \quad$ Li, Feng $^{a} \quad$ Zhang, En $^{a} \quad$ Liu, Hongmin ${ }^{*, a, b}$ \\ ( ${ }^{a}$ School of Pharmaceutical Sciences, Zhengzhou University, Zhengzhou 450001) \\ ( ${ }^{b}$ New Drug Research \& Development Center, Zhengzhou University, Zhengzhou 450001)
}

\begin{abstract}
The oxacephems is unique among $\beta$-lactams. Due to the interesting biological activity and complex synthesis, its derivatives have attracted immense interest for medicinal chemists. Progresses toward the synthesis of oxacephems were reviewed in terms of the key strategies employed.

Keywords oxacephem; oxacepham; asymmitric synthesis; antibiotic
\end{abstract}

$\beta$-内酰胺化合物是抗生素家族使用最广泛的一类 药物, 氧头孢是此类抗生素代表性母核. 氧头孢是由 $\beta$ 内酰胺与六元氧杂环稠并而成, 包括氧头孢烷 (Oxacepham, 1)和氧头狍烯(Oxacephem, 2). 自 1974 年 Wolfe 研究小组 ${ }^{[1 \sim 3]}$ 成功合成氧头孢化合物以来, 其衍 生物优秀的抗菌活性及多个手性中心激发了药物化学 家的浓厚兴趣 ${ }^{[4]}$ (图 1). 氧头孢衍生物对金黄色葡萄球 菌、绿色链球菌等革兰氏阳性菌 ${ }^{[5 \sim 7]}$ 和结肠杆菌、克雷 白氏杆菌属、变形杆菌属等革兰氏阴性菌 ${ }^{[8-10]}$ 有强大的 杀伤能力, 一些氧头孢化合物在临床上作为药物使用. 氟氧头狍(Flomoxef, 3) ${ }^{[11]}$ 和拉氧头孢(Moxalactam, 4) ${ }^{[12]}$ 是治疗脑膜炎及败血症的代表性药物, 可谓抗生素发明 史上璀嗓夺目的明珠.

近年来, 细菌耐药性 ${ }^{[13,14]}$ 及氧头孢烯类抗生素的毒 副作用 ${ }^{[15,16]}$ 越来越制约其发展和临床应用, 合成与研发 新型氧头孢化合物是解决此问题最有效的途径之一. 同 时, 数量浩繁的论文及专利大多阐述氧头孢衍生物的构 效关系或生物活性, 却很少有氧头孢衍生物合成方法学
的综述. 本文从合成方法的角度出发，详细综述了氧头 孢衍生物不对称合成策略的研究进展.

\section{1 合成氧头孢烯骨架的策略}

\section{1 分子内 Witting 反应}

具有显著抗菌、消炎等生物活性的拉氧头孢、氟氧 头孢及其类似物, 均含有光学活性的氧头孢烯骨架. 其 中，1-氧头狍烯母核大多以廉价的 6-氨基青霉烷酸 (6-APA, 13) 为起始原料, 重点集中在 6-位立体性的构筑 二氢噁嗪环(Eq. 1) ${ }^{[17]}$. 通常的方法可以分为三大类: 第 一类是在 6-位引入醚直链后, 经 Witting 反应形成母核. 第二类是在 6-位、7-位噁唑环后, 经 Witting 反应形成母 核. 第三类是在 6-位, 7-位成噁唑环后, 经分子内醚化形 成母核.

6-APA 的噻唑环在氯气条件下容易开环得到氯化 物 15 , 随后在氯化锌的催化下完成 $\beta$-内酰胺环上氯的 取代得到炔丙基醚 16, 产率 39\%. 炔基在 $\mathrm{HgSO}_{4}$ 催化下

\footnotetext{
*E-mail: liuhm@zzu.edn.cn

Received Novembe 3, 2014; revised December 12, 2014; published online January 7, 2015.

Project supported by the National Natural Science Foundation of China (No. 81172937).

国家自然科学基金(No. 81172937)资助项目.
} 
<smiles>CO[C@]1(NC(=O)CSC(F)F)C(=O)N2C(C(=O)O)=C(CSc3nnnn3CCO)CO[C@]2(OC)C1=O</smiles>

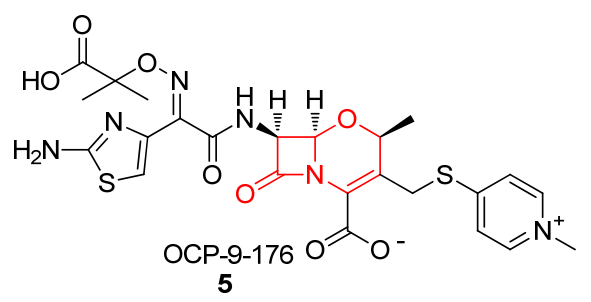<smiles>O=C1C[C@H]2OC[C@@H]3C[C@]3(C(=O)OCc3ccccc3)N12</smiles><smiles>O=C1OC[C@H](c2ccccc2)N1C[C@]1(Cc2ccccc2)C(=O)N2CCCO[C@H]21</smiles>

7<smiles>CCO[C@H](O)[C@H]1CO[C@@H]2CC(=O)N2[C@H]1C(=O)Sc1ccccc1</smiles><smiles>COc1ccc(N2C(=O)[C@H]3OC[C@@H]4CON(C)[C@H]4[C@H]32)cc1</smiles><smiles>COC[C@H]1[C@H]2O[C@H]3OC(C)(C)O[C@@H]3[C@H]2O[C@H]2CC(=O)N21</smiles>

11

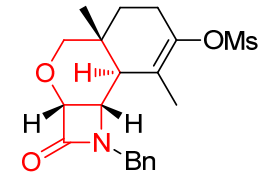

12

图 1 部分有生物活性含氧头孢结构的化合物

Figure 1 Part of bioactive oxacephems and their derivatives

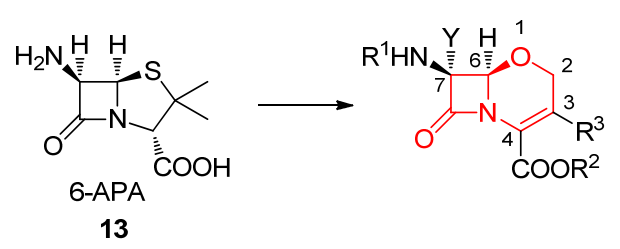

水合成酮，经分子内 Witting 反应得到母核类似物 ${ }^{[18]}$. 由于氯代物 15 是非对映异构体混合物, 导致旋光性变 化, 不能都作为下步反应原料, 故合成 $\mathbf{2 0}$ 的总收率仅为 $26 \%$ (Scheme 1).
1980 年, Kikkawa 等 ${ }^{[19]}$ 采用了新的合成方法, 避免 了 $\beta$-内酰胺手性中心的翻转并有较高收率, 但使用了多 种金属试剂, 条件要求苛刻(Scheme 2).

\section{2 分子内亲核取代}

1980 年, Yoshioka 等 ${ }^{[20]}$ 采用“一锅煮”的方法以 $95 \%$ 的收率得到亚砜酯 26, 合成氧头孢母核 31 的总收率达 $36 \%$. 在此合成路线中，原料 6-APA 的碳原子全部应用 到母核骨架中, 是 “绿色化学” 的明显体现，反应条件 整体温和，适用于工业化生产(Scheme 3).

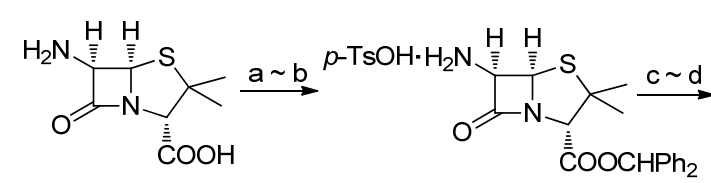

13
14<smiles>CC(C)=C(C(=O)OCc1ccccc1)N1C(=O)[C@H](N)[C@H]1Cl</smiles>

15<smiles>C#CCO[C@H]1[C@H](N)C(=O)N1C(C(=O)O[Ga])=C(C)C</smiles>

16

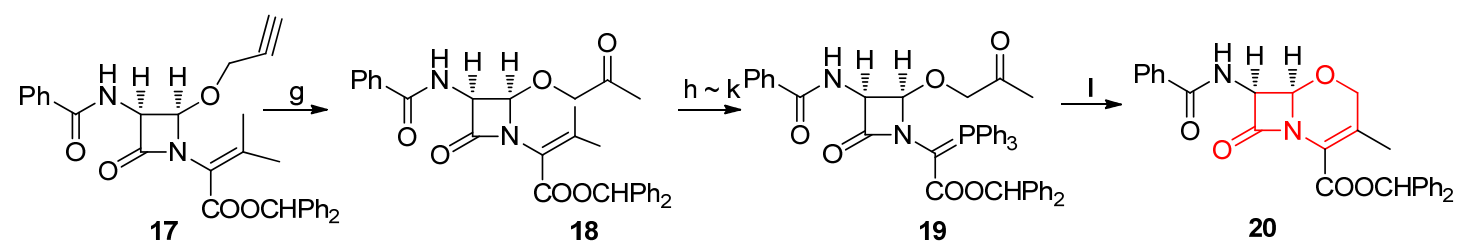

Reagents and conditions: (a) $\mathrm{Ph}_{2} \mathrm{CNNH}_{2}$; (b) $p-\mathrm{TsOH}$; (c) $\mathrm{Cl}_{2}$; (d) $\mathrm{NaHCO}_{3}$; (e) $\mathrm{CH} \equiv \mathrm{CCH}_{2} \mathrm{OH}, \mathrm{ZnCl}_{2}$; (f) $\mathrm{PhCOCl}$; (g) $\mathrm{HgSO}_{4}, \mathrm{H}_{2} \mathrm{O}$; (h) $\mathrm{O}_{3}$; (i) $\mathrm{Zn}, \mathrm{CH}_{3} \mathrm{COOH}$; (j) $\mathrm{SOCl}_{2}, \mathrm{C}_{5} \mathrm{H}_{5} \mathrm{~N}$; (k) $\mathrm{PPh}_{3}$; (l) reflux, dioxane

图式 1 化合物 $\mathbf{2 0}$ 的合成

Scheme 1 Synthesis of compound 20 


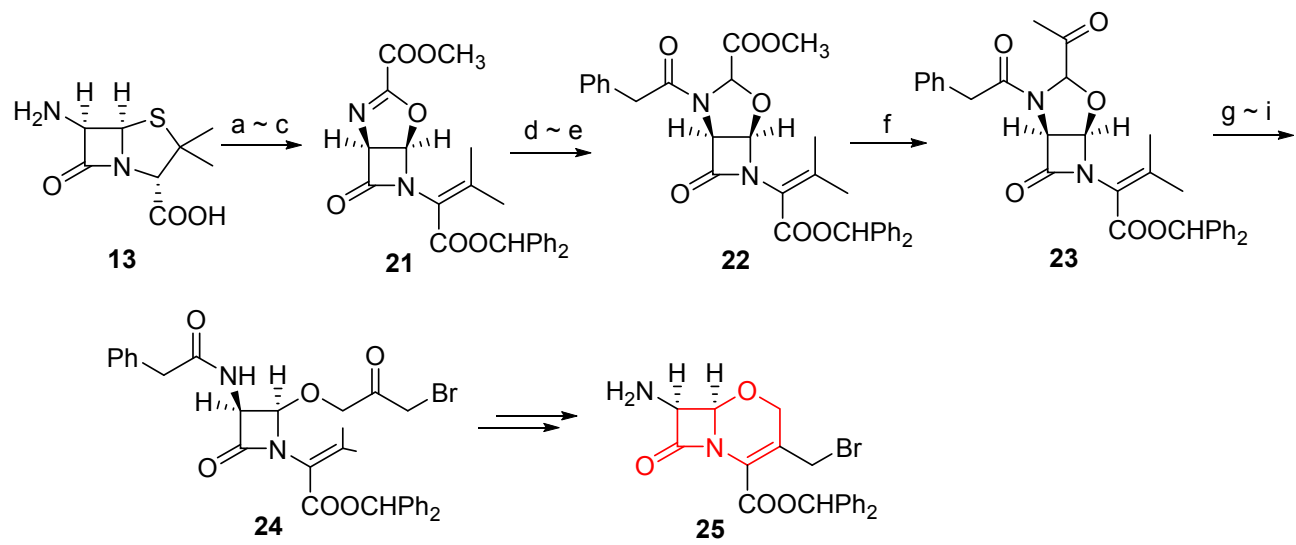

Reagents and conditons: (a) $\mathrm{CH}_{3} \mathrm{OCOCOCl}$; (b) $\mathrm{Cl}_{2}$; (c) $\mathrm{ZnCl}_{2}$, NEt 3 ; (d) $\mathrm{Al}-\mathrm{Hg}$; (e) $\mathrm{PhCH}_{2} \mathrm{COCl}$; (f) $\mathrm{CH}_{3} \mathrm{MgBr}_{\mathrm{NEt}}$; (h) $\mathrm{CuBr} 2, \mathrm{CH}(\mathrm{OEt})_{3}$; (i) $\mathrm{HClO}_{4}$

图式 2 化合物 25 的合成

Scheme 2 Synthesis of compound 25
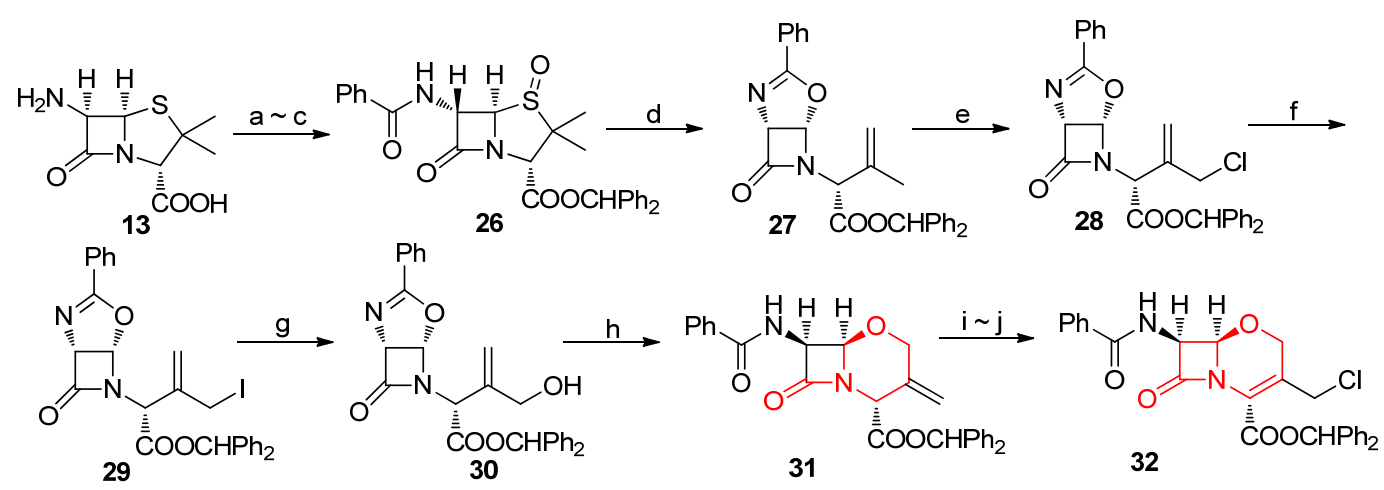

Reagents and conditons: (a) PhCOCl; (b) $\mathrm{CH}_{3} \mathrm{COOOH}$; (c) $\mathrm{Ph}_{2} \mathrm{CNNH}_{2}$; (d) $\mathrm{PPh}_{3}$; (e) $\mathrm{Cl}_{2}$; (f) Nal; (g) $\mathrm{Cu}_{2} \mathrm{O}, \mathrm{DMSO} \mathrm{H}_{2} \mathrm{O}$; (h) $\mathrm{BF}_{3} \cdot \mathrm{Et}_{2} \mathrm{O}$; (i) $\mathrm{Cl}_{2}$; (j) $\mathrm{CH}_{3} \mathrm{OLi}$

图式 3 化合物 32 的合成

Scheme 3 Synthesis of compound 32

\section{3 [2,3]-ס 重排}

Yanagisawa 和 Ando 等 ${ }^{[21]}$ 以 7-氨基头孢烷酸 (7-ACA, 33) 为原料生成亚砜 34, 在加热条件下发生 $[2,3]-\delta$ 重排,与硫醇反应得到二硫化物 36, 经氯气作用, 得到噻唑环. 随后, Eli Lilly 公司 ${ }^{[22]}$ 报道了一个优化的 方法, 亚砜和三苯基磷反应, 加热条件下直接得到噻唑 环，合成 38 的总产率提高了 $20 \%$ (Scheme 4).

\section{2 不对称合成氧头孢烷骨架的策略}

\section{1 亲核取代与分子内闭环}

\section{1 .1 以 4-乙烯氧基氮杂环丁酮为原料}

自 1988 年许多学者报道了 $4 \beta$-甲基取代的氧头孢烯 化合物 OCP-9-176 ${ }^{[23 ~ 25]}, 4 \beta$-甲基的引入显著增强了抗生 素对 $\beta$-内酰胺酶的稳定性而不改变其抗菌活性. 2003 年, Kaluza 等 ${ }^{[26 ~}{ }^{28]}$ 以 4-乙烯氧基氮杂环丁酮 39 为原料, 丁基锂参与, 与溴化物直接反应得到氮烷基化产物, 产
率 79\%. 随后, lewis 酸催化, 经亲核取代进行分子内环 合简单高效的合成出多手性 $4 \beta$-甲基的氧头孢 ${ }^{[29]}$. 不同 酸催化可以得到不同的环合产物 ${ }^{[30]}$, 氧头孢产物为非 对应异构体混合物且不易分离, 很容易氧化转化为相应 的酮(Scheme 5).

5 -氧头孢类 $\beta$-内酰胺酶抑制剂的共同结构特征是氮 杂环丁酮的 C(4)位上有一个烷氧基，最常见的合成策略 是在 C(4)位经亲核取代，随后闭环反应成五元环或六元 环. 此方法缺点是容易手性翻转, 得到非对映异构体或 外消旋体. 2009 年, Koziol 等 ${ }^{[31]}$ 使用联二苯酚类手性试 剂, 立体选择性的合成苯并氧头孢烷 $\mathbf{5 1}, e e$ 值高达 $82 \%$ ～99\%. 4-乙烯氧基氮杂环丁酮与不同的苄溴经亲 核取代得到多种底物, Lewis 酸和手性配体共同催 化 ${ }^{[32 \sim 36]}$ ，闭环形成目标苯并物 51 (Scheme 6). 产物 54 也可以由类似方法环合而成(Scheme 7), 手性配体在对 映选择性中起关键作用且能多次循环使用 ${ }^{[37,38]}$. 


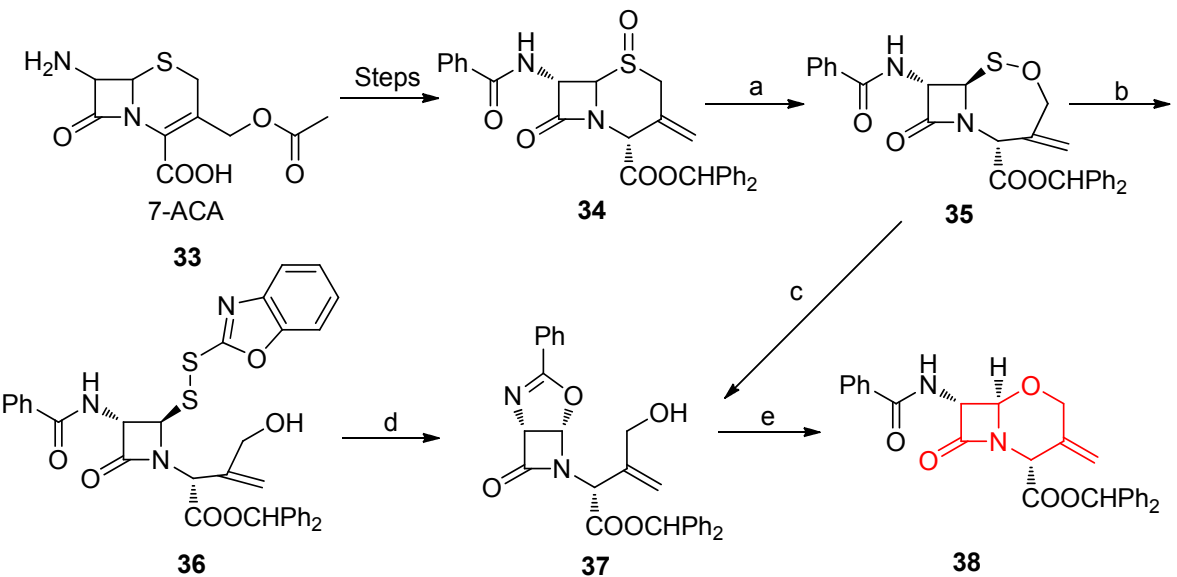

Reagents and conditons: (a) heat; (b) benzo[d]oxazole-2-thiol; (c) $\mathrm{PPh}_{3} ;$ (d) $\mathrm{Cl}_{2}$; (e) $\mathrm{BF}_{3} \cdot \mathrm{Et}_{2} \mathrm{O}$

图式 4 化合物 $\mathbf{3 8}$ 的合成

Scheme 4 Synthesis of compound 38

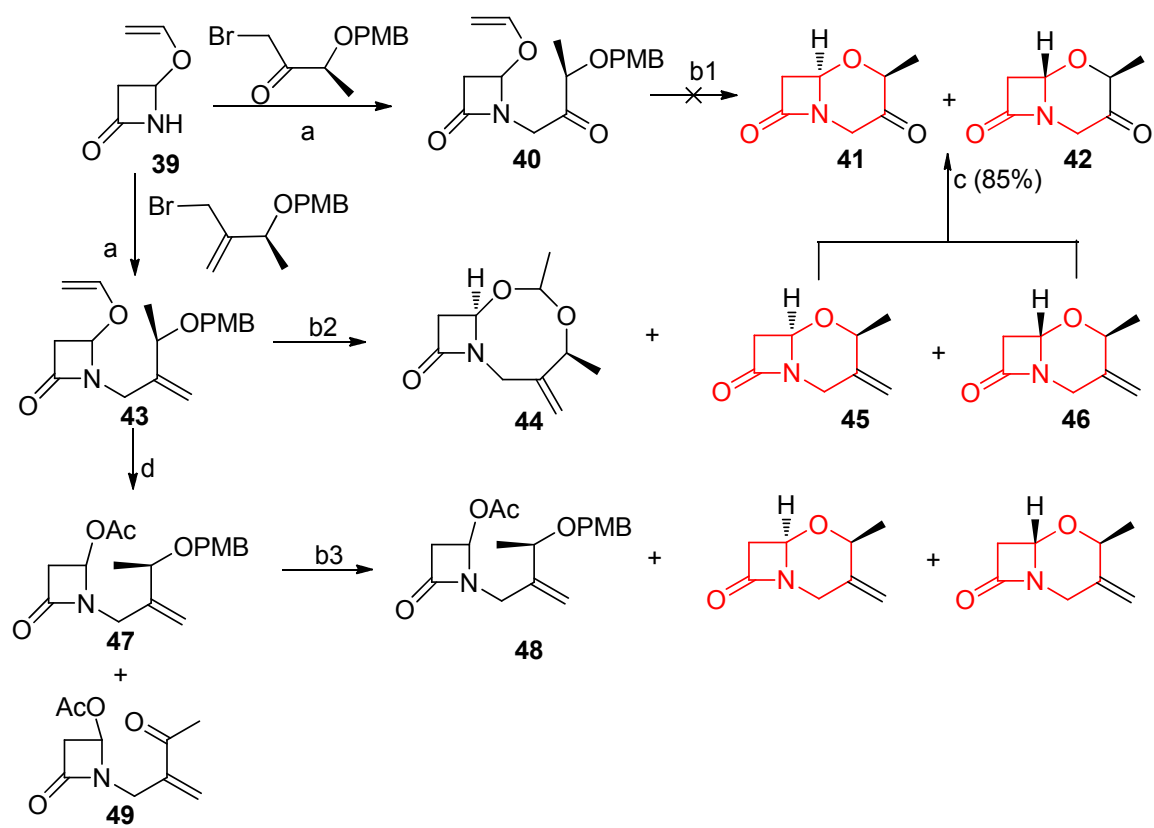

Reagents and conditons: (a) $\mathrm{Bu}_{4} \mathrm{~N}^{+} \mathrm{HSO}_{4}{ }^{-}$, BuLi, THF, 30 min at $-78{ }^{\circ} \mathrm{C}$ then gradually to r.t.; $\left(\mathrm{b}_{1} \sim \mathrm{b}_{3}\right)$ various Lewis acid; (c) $\mathrm{CH}_{2} \mathrm{Cl}_{2} / \mathrm{MeOH}$, $\mathrm{O}_{3}, 10$ min at $-78{ }^{\circ} \mathrm{C}$ then $\mathrm{Me}_{2} \mathrm{~S}$; (d) $\mathrm{PCC} /$ silica gel, $\mathrm{CH}_{2} \mathrm{Cl}_{2}, 6 \mathrm{~h}$, reflux

图式 5 化合物 $\mathbf{4 6}$ 的合成

Scheme 5 Synthesis of compound 46

2002 年, Borsuk 等 ${ }^{[39,40]}$ 以糖片段 $\mathbf{5 5}$ 为底物, 成功合 成出单一的三环手性氧头孢化合物 $\mathbf{5 8}$. 糖与 4-乙烯氧 基氮杂环丁酮的氮原子烷基化, 经分子内取代离去乙烯 氧基，在 $\mathrm{C}(6)$ 位上并成三元环(Scheme 8).

近年来, 固相有机合成(SPOS)在制药及相关工业领 域广泛应用, 能够高效合成先导化合物, 固相合成 $\beta$-内 酰胺酶抑制剂引起了研究人员浓厚的兴趣 ${ }^{[41 ~ 50]}$. 这种 合成策略的基础是形成环状体系，同时产物从固相树脂 中解裂出来. 有机反应中, SPOS 常见于酰胺的形

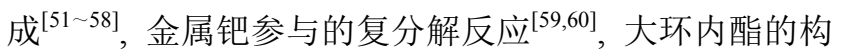

筑 ${ }^{[61,62]}$.

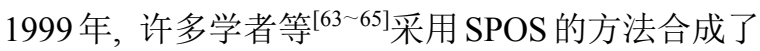
立体单一的四环氧头孢. DMF 作溶剂, 氯甲基树脂 $\mathbf{5 9}$ 与 4-羟基苯甲醛在 $60{ }^{\circ} \mathrm{C}$ 下加热 $24 \mathrm{~h}$, 随后在原甲酸三甲 酯作用下得到二甲基缩醛树脂 61. 该树脂在对甲苯磺 酸吡啶盐(PPTS)参与下, 与二醇成环.二异丁基氢化铝 (DIBAH)开环形成烷氧基, 与 4-乙烯氧基氮杂环丁酮反 应得到氮原子烷基化产物 68 和 69 , 三氟化硼乙醚催化 分子内环合得到六元环, 同时从树脂中分别解裂得到单 一的立体化合物 70 和 71 (Scheme 9). 


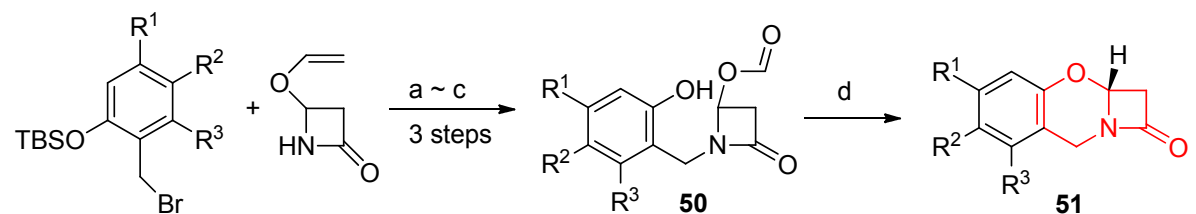

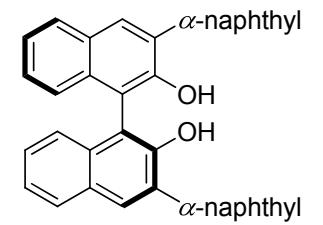

(S)-BINOL

Reagents and conditons: (a) $\mathrm{K}_{2} \mathrm{CO}_{3}$, TBAB, MeCN, reflux, $2 \mathrm{~h}$; (b) TBAF, THF, r.t., $2 \mathrm{~h}$; (c) $\mathrm{O}_{3}, \mathrm{CH}_{2} \mathrm{Cl}_{2},-78{ }^{\circ} \mathrm{C}$ then $\mathrm{Me}{ }_{2} \mathrm{~S}$; (d) (S)-BINOL, $\mathrm{SnCl}_{4}$

图式 6 化合物 51 的合成

Scheme 6 Synthesis of compound 51

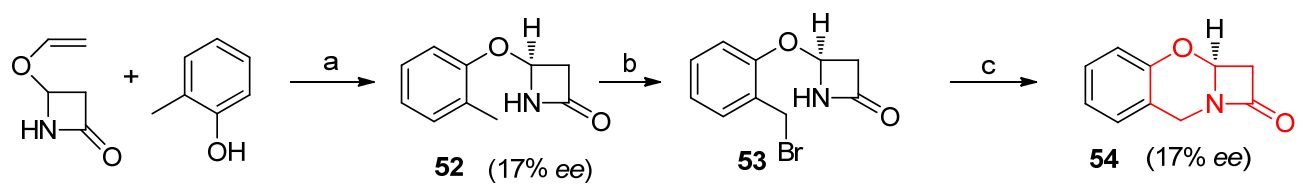

Reagents and conditons: (a) (S)-BINOL, $\mathrm{SnCl}_{4}$; (b) NBS, AIBN, CCl , reflux, 3 h; (c) $\mathrm{K}_{2} \mathrm{CO}_{3}$, TBAB, MeCN, r.t., $12 \mathrm{~h}$

图式 7 化合物 $\mathbf{5 4}$ 的合成

Scheme 7 Synthesis of compound 54

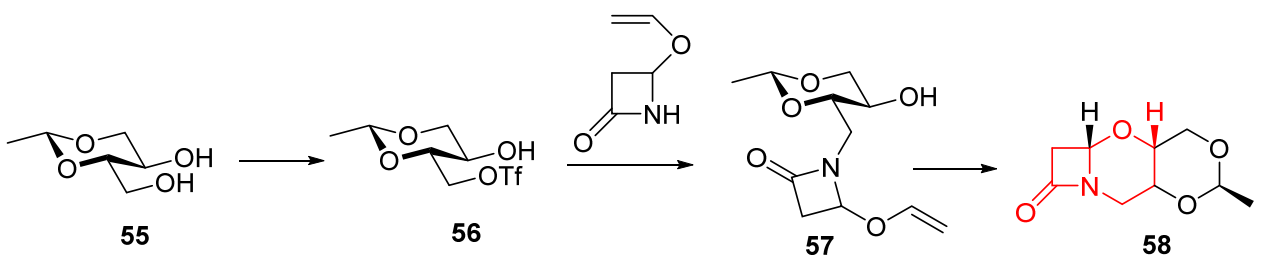

图式 8 化合物 $\mathbf{5 8}$ 的合成

Scheme 8 Synthesis of compound 58

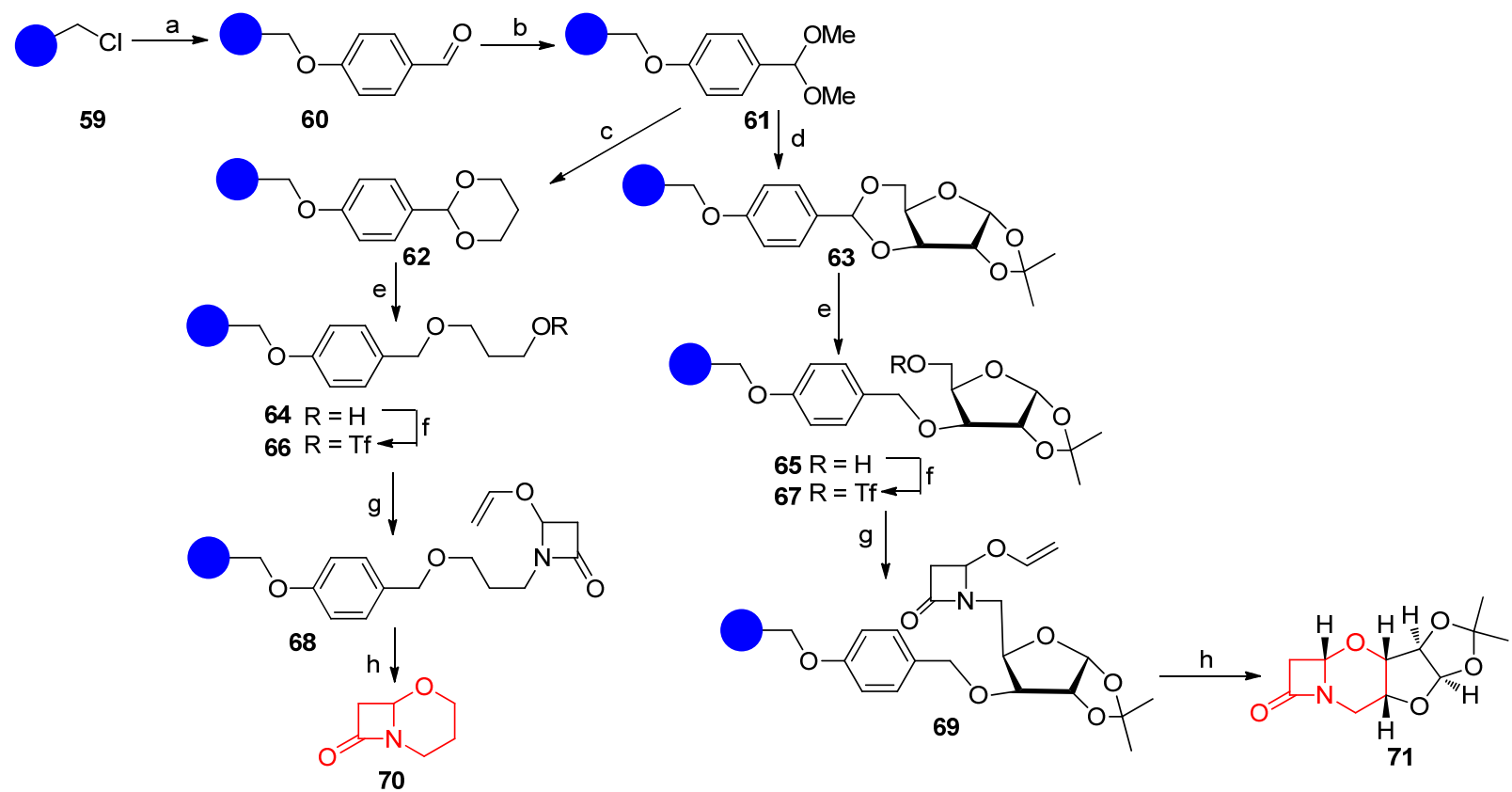

Reagents and conditions: (a) 4-hydroxybenzaldehyde, $\mathrm{NaH}, \mathrm{DMF}, 60{ }^{\circ} \mathrm{C}, 24 \mathrm{~h}$; (b) $\mathrm{HC}(\mathrm{OMe})_{3}$, TsOH, 48 h, r.t.; (c) 1,3-propanediol, pyridinium p-toluenesulfonate, $\mathrm{CH}_{2} \mathrm{Cl}_{2}, 20{ }^{\circ} \mathrm{C}, 24 \mathrm{~h}$; (d) 1,2-O-isopropylidene- $\alpha$ - $D$-xylofuranose, PPTS, $\mathrm{CH}_{2} \mathrm{Cl}_{2}, 20{ }^{\circ} \mathrm{C}, 24 \mathrm{~h}$; (e) DIBAH, $\mathrm{CH}_{2} \mathrm{Cl}_{2},-70{ }^{\circ} \mathrm{C}$ $\rightarrow-20{ }^{\circ} \mathrm{C}, 4 \mathrm{~h}$; (f) $\mathrm{Tf}_{2} \mathrm{O}, 2,6$-lutidine, $\mathrm{CH}_{2} \mathrm{Cl}_{2}, 0{ }^{\circ} \mathrm{C}, 6 \mathrm{~h}$; (g) 4-(vinyloxy)azetidin-2-one, $n$-BuLi, $\mathrm{Bu}_{4} \mathrm{NHSO}_{4}, \mathrm{THF},-70{ }^{\circ} \mathrm{C} \rightarrow-20{ }^{\circ} \mathrm{C}, 12 \mathrm{~h}$; (h) $\mathrm{BF}_{3} \cdot \mathrm{Et}_{2} \mathrm{O}, \mathrm{CH}_{2} \mathrm{Cl}_{2}, 20{ }^{\circ} \mathrm{C}, 3 \mathrm{~h}$

图式 9 化合物 71 的合成

Scheme 9 Synthesis of compound 71 
2.1 .2 以甲酰化氮杂环丁酮或乙酰化氮杂环丁酮为 原料

4-乙烯氧基氮杂环丁酮的乙烯氧基在一定条件下 容易离去, 转化为甲酰氧基或乙酰氧基. 例如, 通过氯 铬酸吡啶嗡盐(PCC)的氧化, 以 $36 \%$ 的产率转化为乙酰 氧基氮杂环丁酮 72; 通过三氯化钉催化或者臭氧解, 以 $82 \%$ 或者 $60 \%$ 的产率转化为甲酰氧基氮杂环丁酮 $\mathbf{7 3}^{[66]}$ (Scheme 10). 乙酰氧基氮杂环丁酮与甲酰氧基氮杂环 丁酮在 $\beta$-内酰胺合成中, 扮演类似的角色. 同时, 4-甲酰 氧基氮杂环丁酮可通过 Baeyer-Villiger 氧化得到 3 位取 代的 4-甲酰氧基氮杂环丁酮类似物 ${ }^{[67 ~ 69]}$.

$N$-烷基化的甲酰氧基氮杂环丁酮能分子内环合得 到氧头孢, 但产率很低. 可以在侧链引入对甲氧基茮基 (PMB)或叔丁基二甲基硅基(TBDMS)等易离去的保护 基, 增强亲核性, 提高分子内环合的产率 (Scheme $11)^{[70]}$.<smiles>CC(=O)OC1CC(=O)N1</smiles><smiles>C=COC1CC(=O)N1</smiles>

Reagents and conditons: (a) $\mathrm{PCC} / \mathrm{SiO}_{2}$; (b) $\mathrm{RuCl}_{3}, \mathrm{NalO}_{4}, \mathrm{O}_{3}$

图式 10 化合物 72 和 73 的合成

Scheme 10 Synthesis of compounds 72 and 73

近年来，手性 Lewis 碱逐渐应用于亲核取代反应. 例如, 金鸡纳碱(Cinchonine)家族在不对称合成中起关 键作用 ${ }^{[71 ~ 76]}$. 2009 年, Koziol 等 ${ }^{[77,78]}$ 以金鸡纳碱作为催 化剂，对映选择性地一步合成了 3,4-苯并氧头孢 82, 同 样克服了产率低的缺点, 产率高达 $92 \%$ (Scheme 12). 不 同的 Lewis 碱催化, 产率与 $e e$ 值差别较大. 当金鸡纳碱 83 的羟基被酰基，甲硅烷基，氨基取代后，不会发生缩 合反应，无法分子内闭环得到氧头孢产物(表 1).
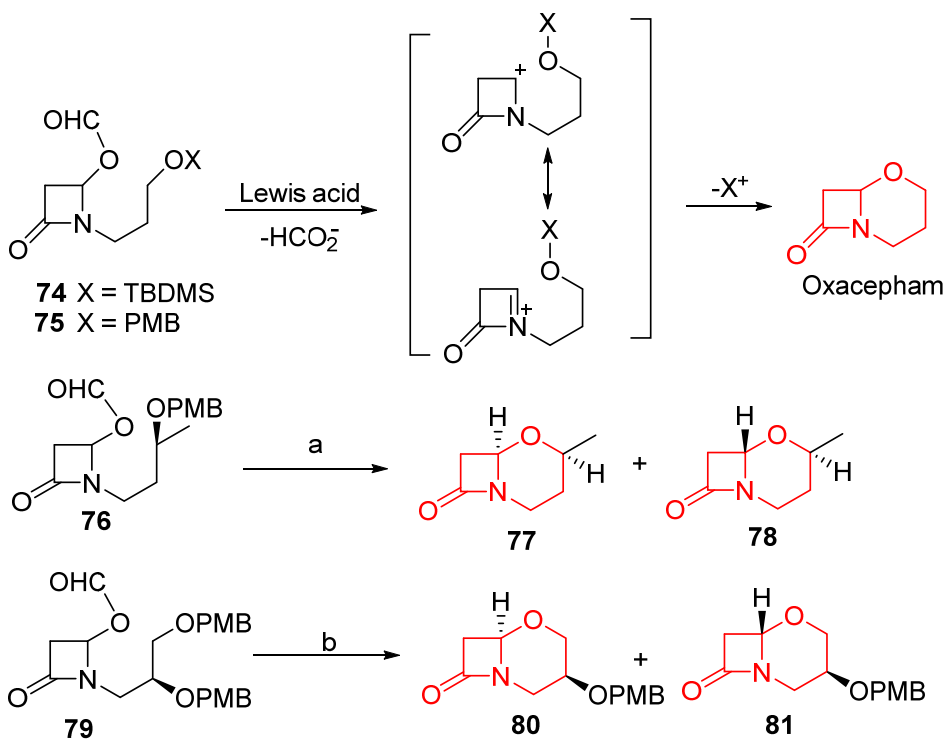

Reagents and conditions: (a) $\mathrm{BF}_{3} \cdot \mathrm{Et}_{2} \mathrm{O}, \mathrm{CH}_{2} \mathrm{Cl}_{2}$; (b) TBAB, $\mathrm{Na}_{2} \mathrm{CO}_{3}, \mathrm{CH}_{3} \mathrm{CN}$

图式 11 化合物 81 的合成

Scheme 11 Synthesis of compound $\mathbf{8 1}$

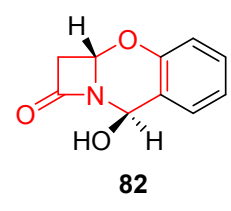

up to $92 \%$ yield up to $48 \%$ ee

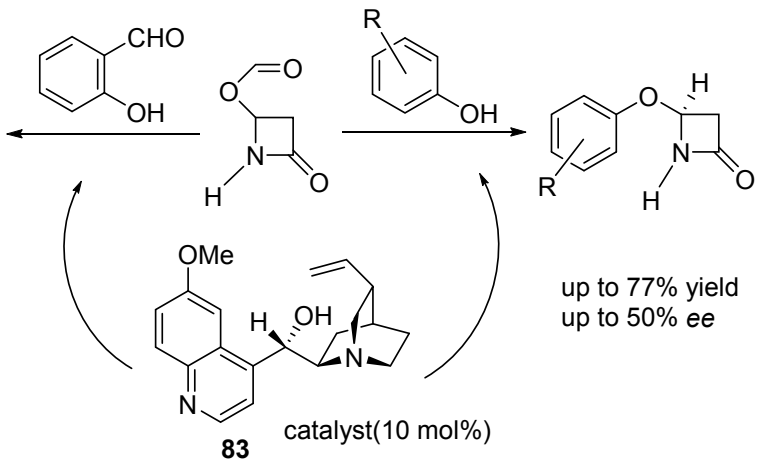

图式 12 化合物 82 的合成

Scheme 12 Synthesis of compound 82 
表 1 探究 0.1 equiv. 手性碱 $\mathbf{A} \sim \mathbf{J}$ 对对映选择性的影响

Table 1 Chiral base evaluation studies for an enantioselective formation in the presence of 0.1 equiv. of chiral Lewis base $\mathbf{A} \sim \mathbf{J}$

\begin{tabular}{cccc}
\hline Entry & Catalyst & Yield $/ \%$ & $e e / \%$ \\
\hline 1 & $\mathbf{A}$ & 77 & 49 \\
$2^{a}$ & $\mathbf{B}$ & 46 & 14 \\
$3^{a}$ & $\mathbf{C}$ & 69 & 14 \\
$4^{a}$ & $\mathbf{D}$ & 63 & 6 \\
5 & $\mathbf{E}$ & 52 & 46 \\
6 & $\mathbf{F}$ & 36 & 16 \\
7 & $\mathbf{G}$ & 0 & \\
8 & $\mathbf{H}$ & 0 & \\
9 & $\mathbf{I}$ & 0 & \\
10 & $\mathbf{J}$ & 0 & \\
\hline
\end{tabular}

${ }^{a}$ Reaction performed in THF.

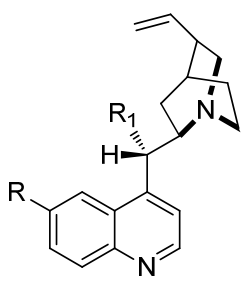

$A \mathrm{R}=\mathrm{OMe}, \mathrm{R}^{1}=\mathrm{OH}$ quinidine $\mathrm{BR}=\mathrm{OMe}$ quinine

$\mathrm{C} R=\mathrm{H}, \mathrm{R}^{1}=\mathrm{OH}$ cinchonine $\quad \mathrm{DR}=\mathrm{H}$ cinchonidine

$F R=O M e, R^{1}=$ NHBOC

$\mathbf{G R}=\mathrm{OMe}, \mathrm{R}^{1}=\mathrm{NH}_{2}$

$\mathrm{HR}=\mathrm{OMe}, \mathrm{R}^{1}=\mathrm{OAC}$

$\mathrm{I} R=\mathrm{OMe}, \mathrm{R}^{1}=\mathrm{OTBS}$

$\mathrm{J} R=\mathrm{OMe}, \mathrm{R}^{1}=\mathrm{OH}$

碱催化体系 $(\mathrm{NaOH}$ 或 EtONa/EtOH)下，4-乙酰氧基 氮杂环丁酮同样可发生亲核取代离去乙酰基团, 缩合反 应闭环形成苯并氧头孢 ${ }^{[79,80]}$ (Scheme 13).

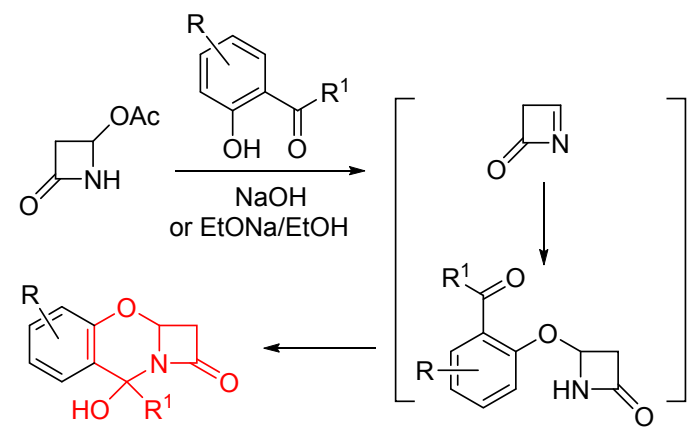

图式 13 3,4-苯并-5-氧头孢烷的合成

Scheme 13 Synthesis of 3,4-benzo-5-oxacephams

\section{$2.2[2+2]$ 环加成}

\subsection{1 以氯磺酰异氯酸酯为原料}

异氧酸酯一直广泛应用于有机合成 ${ }^{[81 ~ 83]}$ 和候选药 物结构优化 ${ }^{[84 ~ 86]}$ 中, 这类化合物也被用来构筑内酰胺 环. 1985 年, Buynak 和 Kaluza 等 ${ }^{[87 ~ 92]}$ 以累积二烯烃为底 物, 与氯磺酰异氧酸酯(CSI) $[2+2]$ 环加成得到 $\beta$-内酰 胺. 随后, 许多学者等 ${ }^{[93 ~ 96]}$ 在糖片段 84 上引入环外双 键, 与 CSI 环加成得到绝对构型的 $\beta$-内酰胺 86 , 经氮原<smiles>CCC1CN2CCC1CC2C(Oc1ccc(OC(c2ccnc3ccc(OC)cc23)C2CC3CCN2C3)cc1)c1ccnc2ccc(OC)cc12</smiles>

E

子分子内烷基化闭环得到四环氧头孢(Scheme 14). 不 同的糖片段作为起始原料(Starting material)可以得到不 同的多环体系类似物(图 2), 产率较高, 方法成熟. 2004 年, Lysek 等 ${ }^{[97 \sim 99]}$ 利用烷基磺酰基作为 linker, 实现了聚 苯乙烯树脂和含环外双键的糖片段的连接, 通过 $[2+2]$ 环加成和分子内烷基化, 脱去树脂, 得到四环氧头孢. 2014 年, Lee 等 ${ }^{[100]}$ 采用相同的加成方法以 1,4-环已二烯 为原料与 CSI, 在氢氧化钠参与下合成双环 $\beta$-内酰胺.
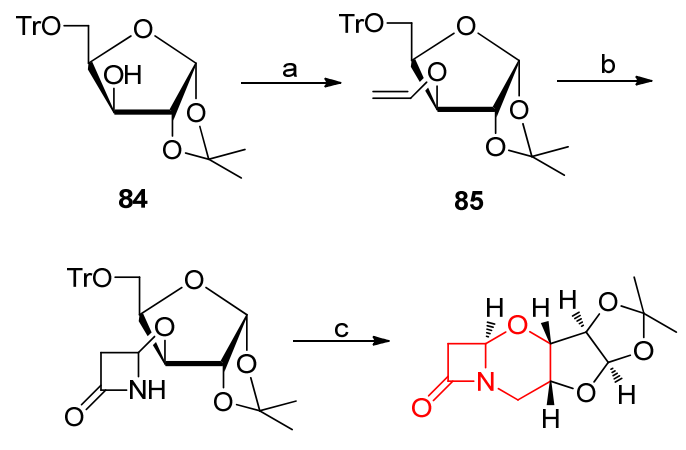

86

87

Reagents and conditions: (a) 1) $\mathrm{NaH}, \mathrm{DMF}, \mathrm{CH}_{2} \mathrm{CHBr}$; 2) $t$-BuOK/ DMSO; (b) CSI, $\mathrm{Na}_{2} \mathrm{CO}_{3} /$ Red-Al; (c) 1) TsOH/MeOH; 2) TsCl/Py.; 3) $\mathrm{K}_{2} \mathrm{CO}_{3}, \mathrm{TBAB} / \mathrm{CH}_{3} \mathrm{CN}$

图式 14 化合物 87 的合成

Scheme 14 Synthesis of compound 87 


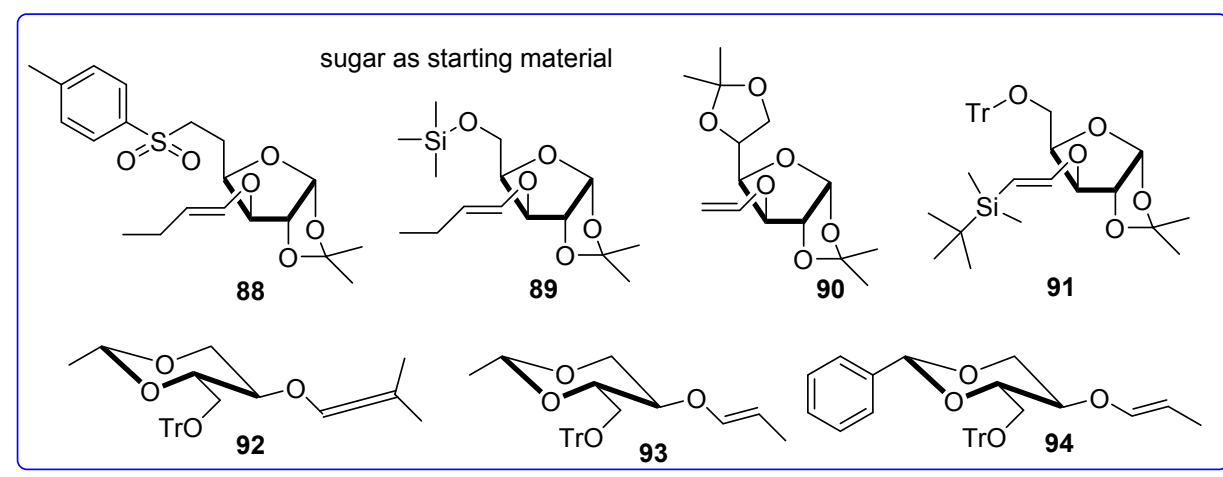

React with CSI

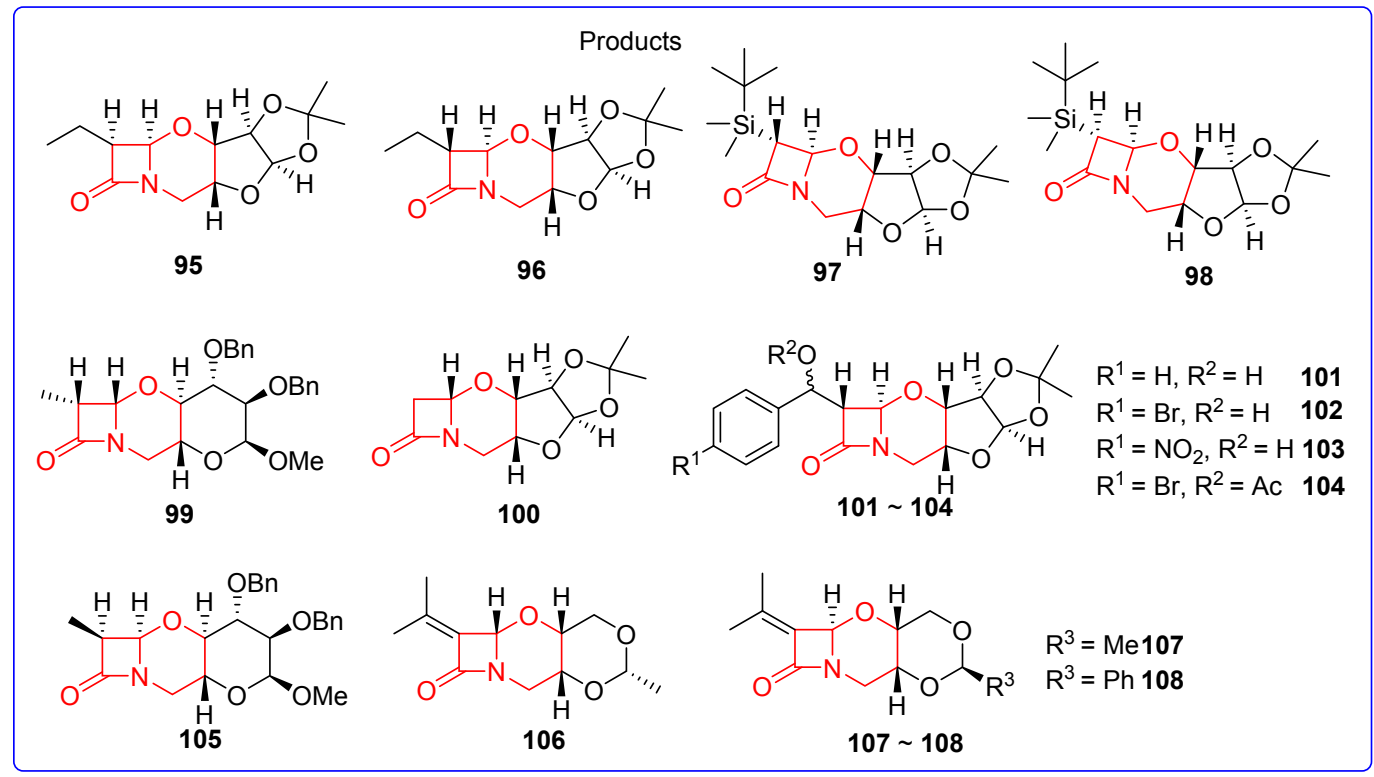

图 2 糖为原料合成手性氧头孢衍生物

Figure 2 Synthesis oxacephems derivatives from sugars as starting material

1987 年, Buynak 等 ${ }^{[101]}$ 基于 CSI 的[2+2]环加成, 得 到一系列 3 位亚烷基取代的氮杂环丁酮衍生物. 这种结 构修饰的策略有两方面的价值: (1)环外双键广泛存在于 抗生素和 $\beta$-内酰胺酶抑制剂 ${ }^{[102]}$ 中. 例如, asparenomy$\operatorname{cins}^{[103 \sim 106]}$, alkylidenepenicillins ${ }^{[107,108]}$, alkylidenecephalosporins ${ }^{[109]}$ 等抗生素, 环外双键对其生物活性均产生 重要影响 ${ }^{[110]}$. (2)环外双键可以转化为异丙基、羟基异丙 基、卤基、甲酰基等活性官能团. 含累积二烯烃结构的 糖片段 109 通过环加成和分子内环合可以得到含环外双 键的氧头狍 111 和 112, 随后经一系列的反应, 氧头狍结 构中引入羟基、卤基、羰基等容易修饰的活性位点, 为 丰富活性氧头孢化合物的种类提供了可能(Scheme 15).

\subsection{2 以环状亚胺为原料}

近年来, 以烯酮和亚胺为底物, 经 $[2+2]$ 环加成合 成手性 $\beta$-内酰胺 ${ }^{[111 ~ 113]}$ 的策略被广泛用于合成手性 $\beta$ 内酰胺 ${ }^{[114 \sim 120]}$. 2005 年, Lei 等 ${ }^{[121]}$ 以环状亚胺 126 为原
料, 三乙胺催化下, 甲苯做溶剂, 与苯氧乙酰氯环加成 得到氧头孢 128, 产率 $40 \% .2011$ 年, $X u$ 等 ${ }^{[122]}$ 在光照射 下, 环状亚胺与重氮乙酸酯 130 反应得到氧头孢类似物 和其他多环手性 $\beta$-内酰胺(Scheme 16).

\section{2 .3 以邻氨基苯酯为原料全合成氧头狍}

1984 年, Ongania 等 ${ }^{[123]}$ 以邻氨基苯酯 131 为原料, 三氯氧磷催化下，与 $N, N$-二甲基甲酰胺(DMF)反应生成 亚胺, $\mathrm{H}_{2} \mathrm{~S}$ 参与, 引入甲硫基. 亚胺与氯乙酰氯[2+2]环 加成得到 $\beta$-内酰胺 135, $\mathrm{Bu}_{3} \mathrm{SnH}$ 或 $\mathrm{AIBN}$ 催化, 脱去卤 原子, $\mathrm{Pd} / \mathrm{C}$ 催化脱除酯基. 氧化录氧化环合得到终产物 139, 副产物少, 产率高, 为工业化大规模生产提供了一 种新的思路(Scheme 17).

\subsection{Fischer Carbene 的光解反应}

含有 $\left[\mathrm{C}(\mathrm{H}) \mathrm{NR}_{2}\right]$ 基团的铬卡宾复合物(Fischer Carbene), 可以由 Vilsmeir's 盐与 $\mathrm{Cr}(\mathrm{CO})_{5}{ }^{2-}$ 合成 ${ }^{[123,124]}$, 其 


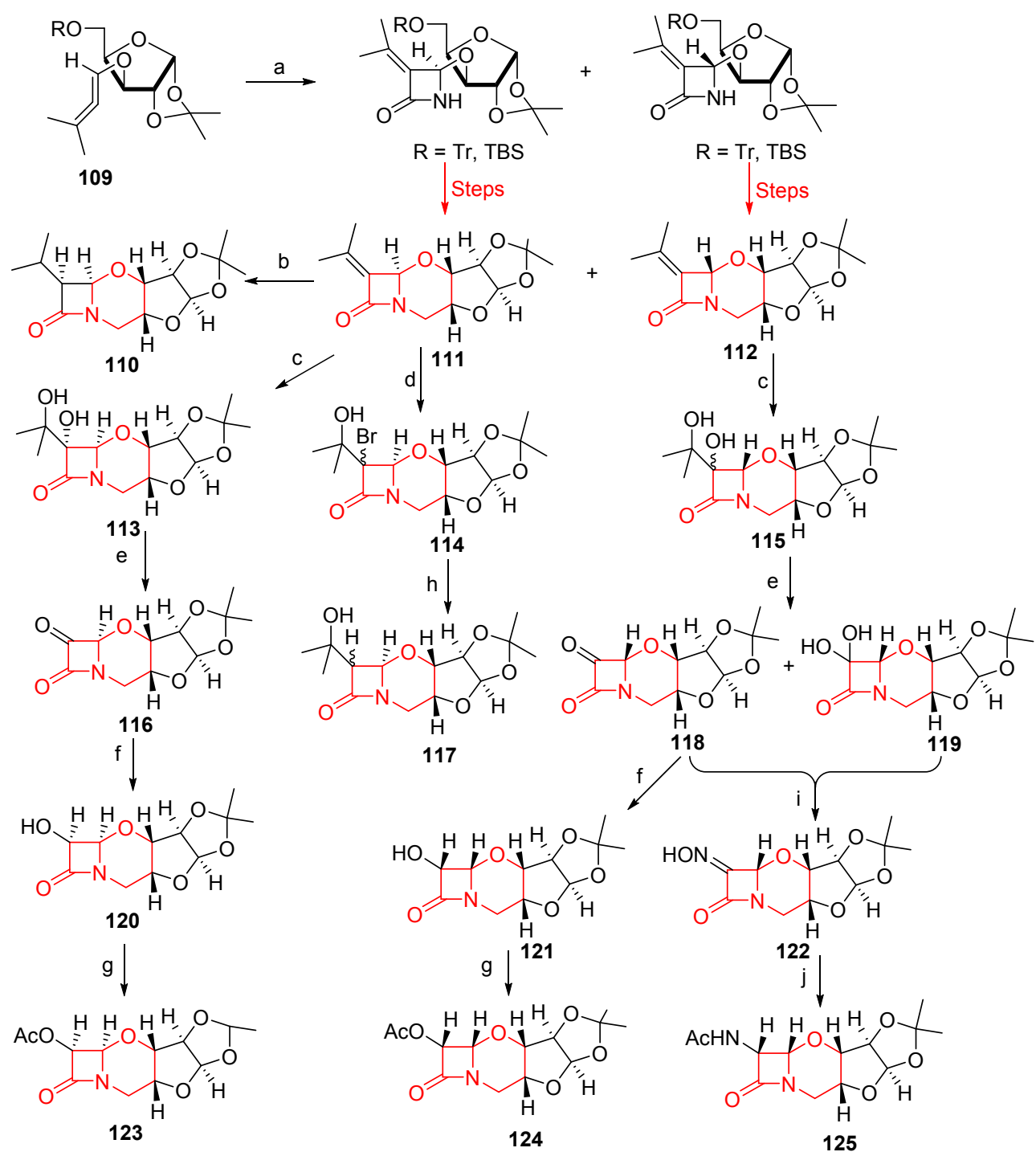

Reagents and conditions: (a) 1) CSI, $\mathrm{Na}_{2} \mathrm{CO}_{3}$; 2) Red-Al; (b) $\mathrm{H}_{2} / \mathrm{PtO}_{2}, \mathrm{MeOH}$; (c) RuCl $3 / \mathrm{NalO}_{4}, \mathrm{H}_{2} \mathrm{O} / \mathrm{CH}_{3} \mathrm{CN} / \mathrm{CCl}_{4}$; (d) NBS, DMSO; (e) $\mathrm{H}_{6}$ IO AcOEt; (f) $\mathrm{NaBH}_{4}, \mathrm{MeOH}$; (g) AcOH; (h) $\mathrm{Bn}_{3} \mathrm{SnH}$, AlBN, toluene, $100{ }^{\circ} \mathrm{C}$; (i) $\mathrm{NH}_{2} \mathrm{OH} \cdot \mathrm{HCl}$, Py; (j) 1) $\mathrm{AcONa}, \mathrm{Ac}_{2} \mathrm{O}, \mathrm{AcOEt}$ 2) PtO ${ }_{2}, \mathrm{H}_{2}$

图式 15 化合物 125 的合成

Scheme 15 Synthesis of compound $\mathbf{1 2 5}$

对空气稳定，可以抵抗亲核试剂的进攻。近年来， Hegedus 等 ${ }^{[125,126]}$ 研究的 Fischer Carbene 的光解反应被 广泛用于有机合成中，用以制备环丁酮 ${ }^{[126 ~ 128] 、 ~} \beta$-内酯、 $\gamma$-内酯 ${ }^{[129]}$ 、氨基酸 ${ }^{[130]}$ 、丙二烯、肽、二环[4.2.1]壬酮衍 生物、2-丁烯-1,4-二酮, 多环芳香化合物. 光化学反应中

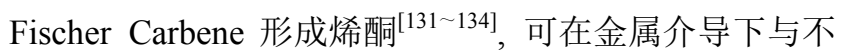
饱和化合物发生亲核加成或环加成反应，条件非常温和 (Scheme 18).

1987 年, Borel 等 ${ }^{[135]}$ 采用此方法, 以 Fischer Carbene 140 和环状亚胺 141 反应得到单一的手性氧头孢 143 , 产 率 $81 \%$, 随后在氢气下, $\mathrm{Pd} / \mathrm{C}$ 催化脱苠基得到氨基氧头 孢 121，产率 94\%. 1993 年, Colson 等 ${ }^{[136]}$ 在此基础上，利 用铬卡宾的光解制备氧头孢中间体 147 , 经过一系列的
反应得到 $\alpha$-烷基- $\alpha$-氨基酸(Scheme 19).

\section{4 钯催化的 Ene-Halogenocyclization}

以氮杂环丁酮为原料合成良好生物活性的双环 $\beta$ 内酰胺衍生物, 方法很多. 1986 年, Mori 等 ${ }^{[137]}$ 通过钯催 化有效合成了双环 $\beta$-内酰胺，4-乙酰基氮杂环丁酮作为 原料转化为氯化物 152, 在四三苯基磷钯和六甲基磷酰 三胺(HMPA)催化下，以 $35 \%$ 产率得到两个七元环的异 构体 153 和 154, 然后分别与 1,8-二氮杂二环十一碳-7烯(DBU)作用，发生 Ene-Halogenocyclization 反应，得到 单一的氧头孢 155 和 156 (Scheme 20). 近年来, 有机合 成中采用类似的手段, 进行钯催化环合均得到了理想化 合物 ${ }^{[138,139]}$. 


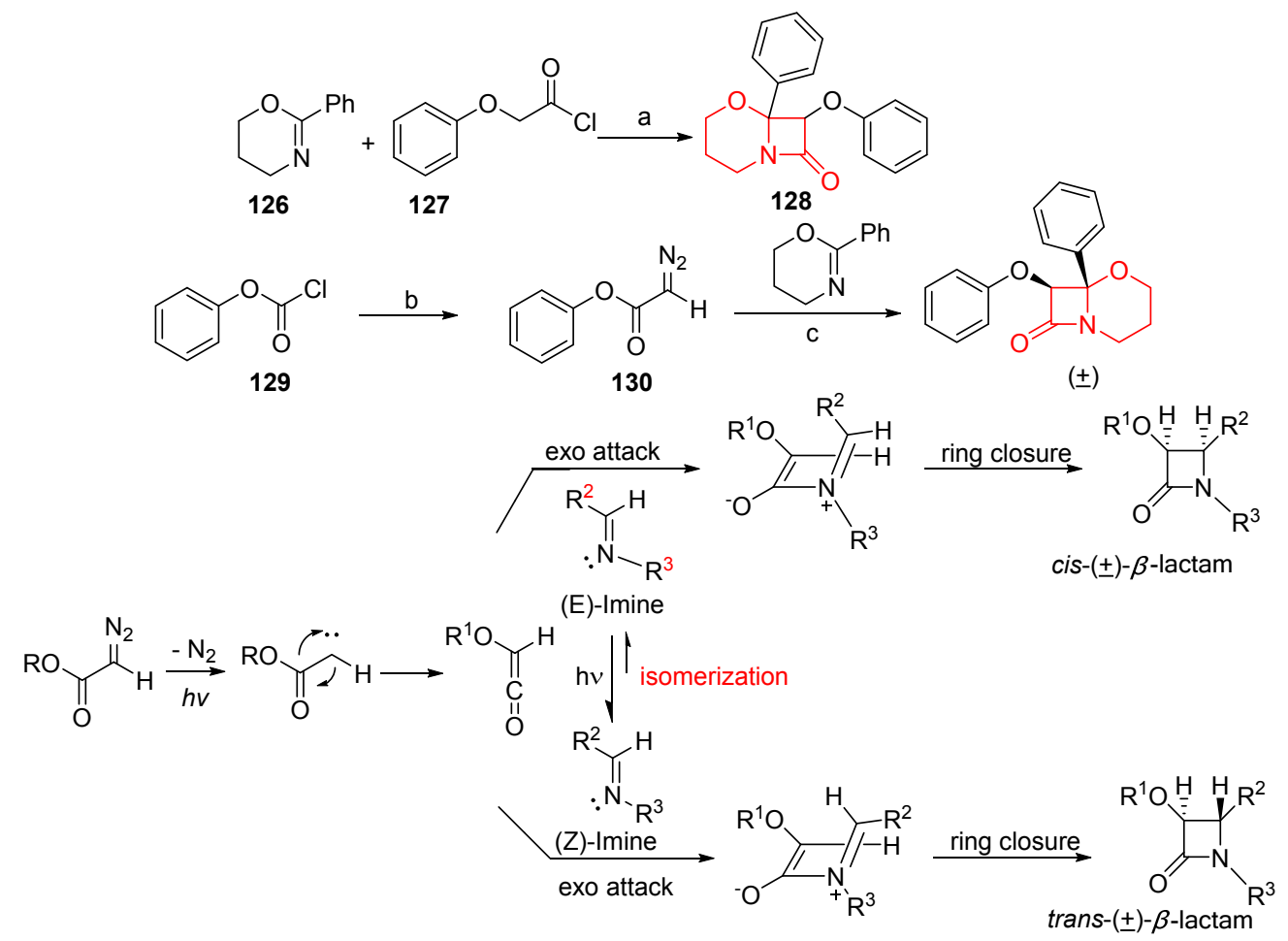

Reagents and conditions: (a) $\mathrm{Et}_{3} \mathrm{~N}$, toluene; (b) $\mathrm{CH}_{2} \mathrm{~N}_{2}$; (c) hv, $\mathrm{CH}_{2} \mathrm{Cl}_{2}$, r.t., 6 h

图式 16 光照下重氮乙酸酯合成氧头孢烷

Scheme 16 Synthesis of oxacephams using diazoacetate esters under photoirradiation<smiles>[R]c1cccc(C(=O)OCc2ccc(Br)cc2)c1NC(=S)Nc1ccc(C(=O)OCc2ccccc2)c(/N=C/N(C)C)c1[R]</smiles>

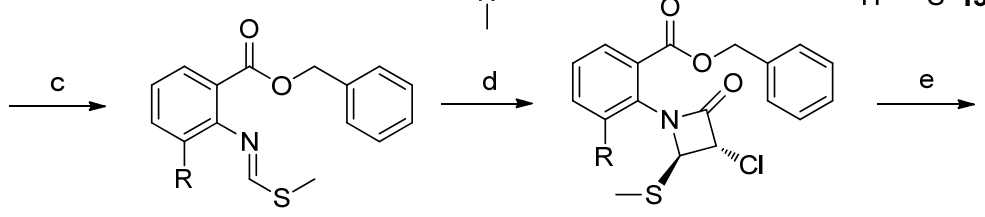

134<smiles>[R]c1cccc(C(=O)OCc2ccccc2)c1N1C[C@@H](SC)CC1=O</smiles><smiles>[3H]C</smiles><smiles>[R]c1cccc(C(=O)O)c1N1C(=O)C(C)CC1SC</smiles>

135

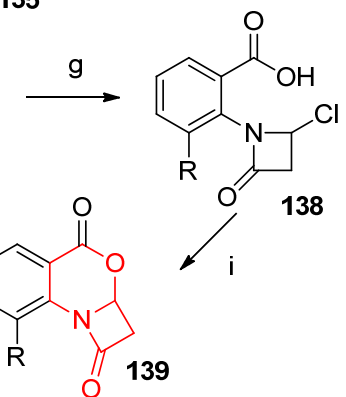

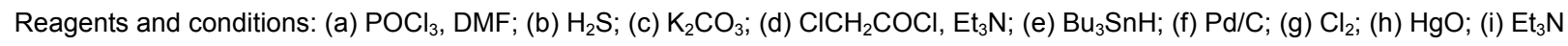

图式 17 化合物 139 的合成

Scheme 17 Synthesis of compound 139

\section{$2.5[3,3]-\delta$ 重排与 Diels-Alder 的串联反应}

$\alpha$-累积二烯醇广泛用于天然产物和候选药物的不 对称合成, 其可以由醛与取代的溴丙炔在水介质中制
备 ${ }^{[140,141]} .2005$ 年, Alcaide 等 ${ }^{[142 ~ 149]}$ 以 $\alpha$-累积二烯醇- $\beta$ 内酰胺 158 或 161 为原料, 甲磺酰氯/三乙胺作用下, 由 单环体系稠环得到三环体系(Scheme 21). 


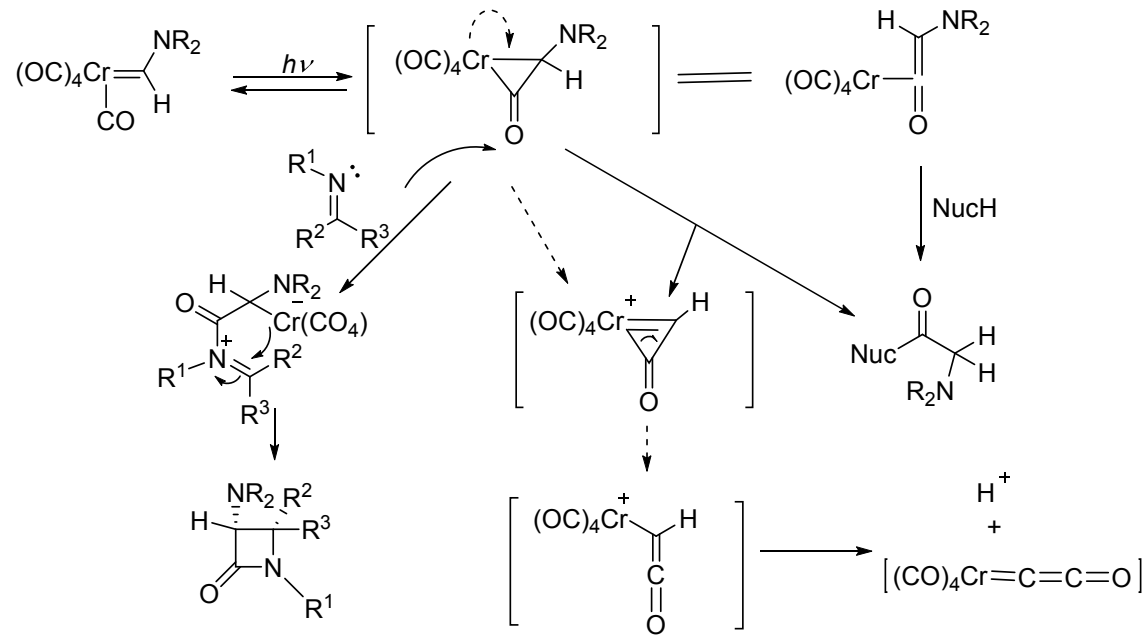

图式 18 铬卡宾与亚胺的光解反应机理

Scheme 18 The mechanism of the photolytic reaction of chromium-carbene with imines

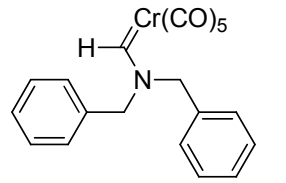

140

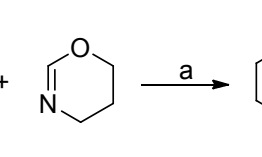

141

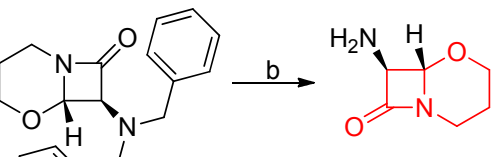

142
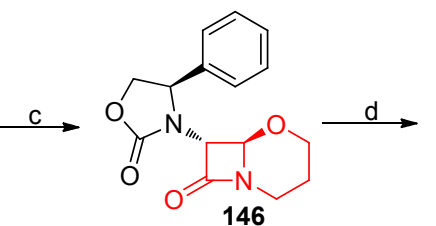

(R)-1

145 $96 \%$ yield $>97 \%$ de

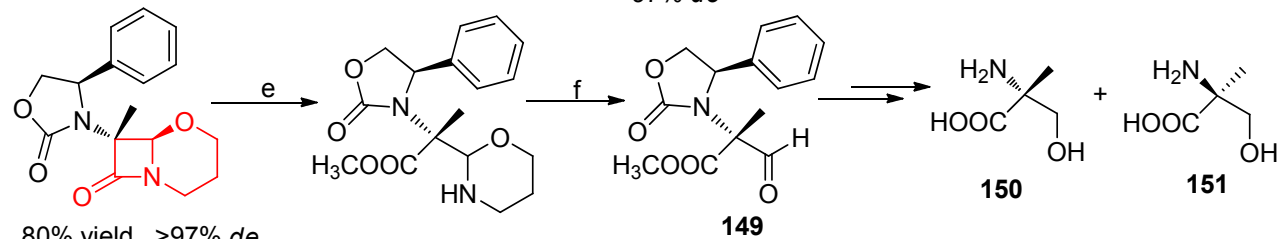

$80 \%$ yield, $>97 \%$ de

147

148

$50 \% \sim 55 \%$ yield

Reagents and conditions: (a) $\mathrm{hv}, \mathrm{Et}_{2} \mathrm{O}, \mathrm{CO}, 81 \%$ yield; (b) $\mathrm{H}_{2}, \mathrm{Pd} / \mathrm{C}, 81 \%$ yield; (c) 1 ) $0.2 \mathrm{~mol} / \mathrm{L} \mathrm{HCl}, \mathrm{THF}, 25{ }^{\circ} \mathrm{C}$; 2 ) Triphosgene, $i-\mathrm{Pr}_{2} \mathrm{NEt}$, $\mathrm{CH}_{2} \mathrm{Cl}_{2}, 0{ }^{\circ} \mathrm{C}$; (d) 1$) \mathrm{KHMDS}$, THF, $-78{ }^{\circ} \mathrm{C}$; 2) DMF; 3) $\mathrm{CH}_{3} \mathrm{l},-78{ }^{\circ} \mathrm{C}$; 4 ) aq. $\mathrm{NH}_{4} \mathrm{Cl}$; (e) $\mathrm{HCl}$; (f) $1 \mathrm{~mol} / \mathrm{L} \mathrm{HCl}, \mathrm{THF}, 48 \mathrm{~h}, 25{ }^{\circ} \mathrm{C}$

图式 19 化合物 147 的合成

Scheme 19 Synthesis of compound 147

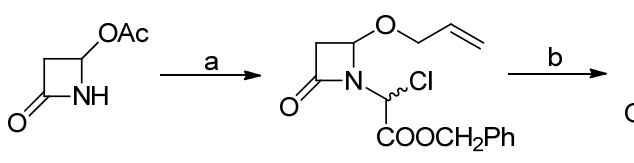

152

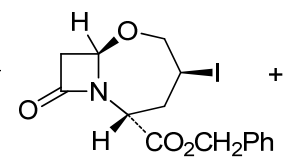

153

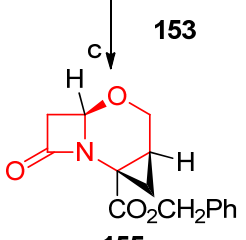

155
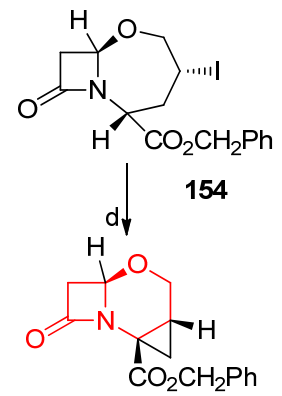

156

Reagents and conditions: (a) $\mathrm{CH}_{2} \mathrm{CHCH}_{2} \mathrm{OH}, \mathrm{PhCH}_{2} \mathrm{O}_{2} \mathrm{CCHO}$; (b) $\mathrm{Pd}\left(\mathrm{PPh}_{3}\right)_{4}, \mathrm{HMPA}, \mathrm{KI}$; (c) DBU, 15 min, 85\% yield; (d) DBU, 48 h, $80 \%$ yield

图式 20 化合物 155 和 156 的合成

Scheme 20 Synthesis of compound 155 and 156 


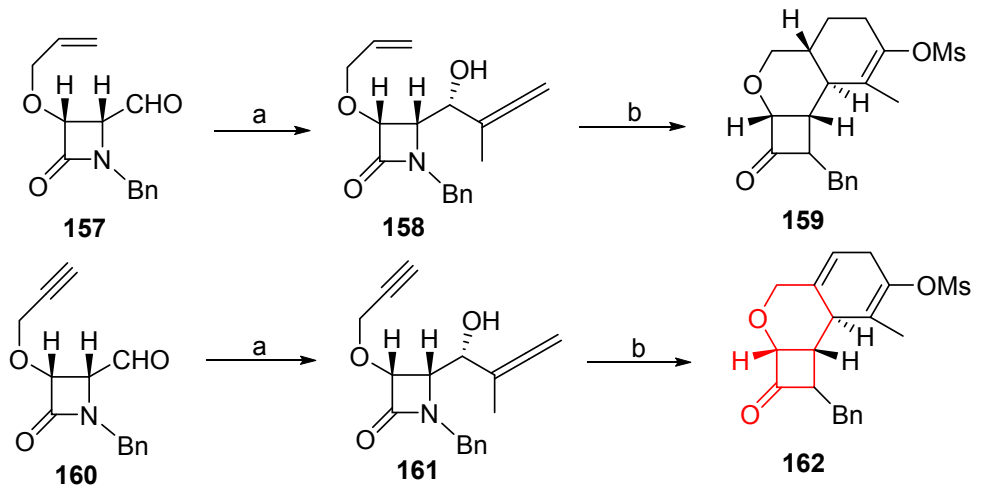

Reagents and conditions: (a) $\mathrm{CH}_{3} \mathrm{CCCH}_{2} \mathrm{Br}$, THF/NH${ }_{4} \mathrm{Cl}$ (aq. sat); (b) $\mathrm{CH}_{3} \mathrm{SO}_{2} \mathrm{Cl}, \mathrm{Et}{ }_{3} \mathrm{~N}$, toluene, sealed tube, $190{ }^{\circ} \mathrm{C}$

图式 21 化合物 162 的合成

Scheme 21 Synthesis of compound 162

上述复杂分子的设计用到串联反应(Tandem reaction)的理念，其符合原子经济性，能节省时间和反应成 本，这种多步、一锅煮的合成策略是以后的发展趋势. $\alpha-$ 累积二烯醇- $\beta$-内酰胺在甲磺酰氯/三乙胺作用下, 首先 发生 $[3,3]-\delta$ 重排, 形成共轭二烯烃. 此共轭二烯烃作为 原料, 相同条件下发生分子内 Diels-Alder 反应, 形成三 元环 ${ }^{[150,151]}$ (Scheme 22).

\section{3 结语与展望}

抗生素、尤其是 $\beta$-内酰胺类抗生素的合成是医药研 发最重要的内容之一, 是生命科学与自然科学完美交叉 的体现，尽管日本盐野义制药株式会社在 20 世纪 80 年
代首先研发并生产了拉氧头孢，但新的合成方法与优良 活性的氧头狍化合物仍不断地被开发出来，合成的 策略不断地得到改进和创新.

本文对近年来活性较好的新颖氧头孢类似物不对 称合成路线进行了简单概括，希望能为不对称合成此类 化合物提供一些依据, 并由此开拓出更加实用高效的合 成方法. 合成氧头孢仍存在很多困难，诸如反应条件苛 刻、

贵重金属有毒试剂的使用、路线长、手性难以控制 等，所以寻求条件温和、路线简短的不对称合成方法及 设计更加多样化的活性化合物必将是未来的一种趋势.
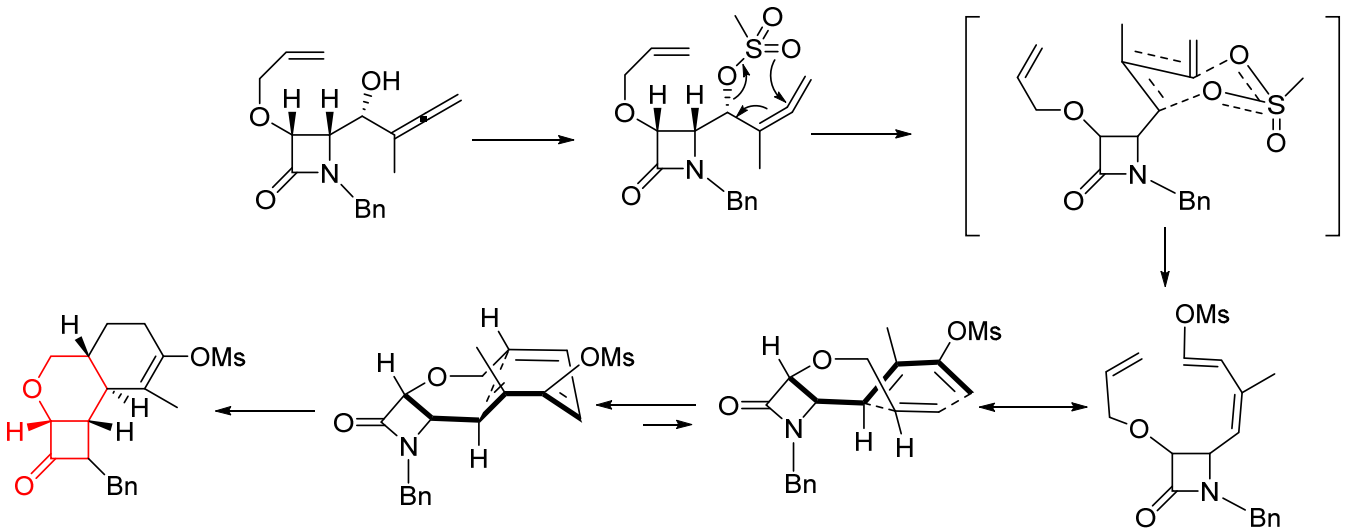

图式 22 合成化合物 159 的串联反应机理

Scheme 22 The mechanism for the tandem reaction to synthesize compound $\mathbf{1 5 9}$

\section{References}

[1] Otsuka, H.; Nagata, W.; Yoshioka, M.; Narisada, M.; Yoshida, T.; Harada, Y.; Yamada, H. Med. Res. Rev. 1981, 217.

[2] Shibahara, S.; Okonogi, T.; Murai, Y.; Kudo, K.; Yoshida, T.; Kondo, S.; Christensen, B. G. J. Antibiot. 1988, 41, 1154.

[3] Zbigniew, K.; Arkadiusz, K.; Krzysztof, L.; Kinga, S.; Beata, S.; Marek, C. Tetrahedron 2003, 59, 5893.

[4] Li, Y.-F.; Hu, J. Y.; Liu, Q. B.; Zhang, Z. H.; Zhang, F. J. Chin. J. Org. Chem. 2008, 28, 1 (in Chinese).
(李耀峰, 胡进勇, 刘庆涁, 张占辉, 张福军, 有机化学, 2008, 28,1 .)

[5] Strieker, M.; Kopp, F.; Mahlert, C.; Essen, L.-O. ACS Chem. Biol. 2007, 2, 187.

[6] Pieri, C.; Borselli, D.; Giorgio, C. D.; Meo, M. D.; Bolla, J.-M.; Vidal, N.; Combes, S.; Brunel, J. M. J. Med. Chem. 2014, 57, 4263.

[7] Omar, M.-C.; Reynolds, R.-C. ACS Comb. Sci. 2014, 16, 232.

[8] Nie, S. Y.; Yu, B. J. Am. Chem. Soc. 2014, 136, 4157.

[9] Roberts, D. M.; Personne, Y.; Ollinger, J.; Parish, T. Future Microbiol. 2013, 8, 621 . 
[10] Frees, D.; Qazi, S. N. A.; Hill, P. J.; Ingmer, H. Mol. Microbiol. 2003, 48, 1565.

[11] Tsubouchi, H.; Tsuji, K.; Yasumura, K.; Matsumoto, M.; Shitsuta, T.; Ishikawa, H. J. Med. Chem. 1995, 38, 2152.

[12] Narisada, M.; Yoshida, T.; Ohtani, M.; Ezumi, K.; Takasuka, M. J. Med. Chem. 1983, 26, 1577.

[13] Christopher, T.; Gerry, W. Chem. Rev. 2005, 105.

[14] Zhang, Q. C.; Robin, K.; Liao, D.; Lambert, G.; Austin, R. H. Mol. Pharm. 2011, 8, 2063.

[15] Hurley, L. H.; Reck, T.; Thurston, D. E.; Langly, D. R. Chem. Res. Toxicol. 1988, 1, 258.

[16] Zhang, J. P.; Qian, J. Q.; Tong, J. W.; Zhang, D. S.; Hu, C. Q. Chem. Res.. Toxicol. 2013, 26, 1168.

[17] Narisada, M.; Tsuji, T. Recent Prog. Chem. Synth. Antibiot. 1990, 706.

[18] Narisada, M.; Onoue, H.; Nagata, W. Heterocycles 1977, 7, 839.

[19] Kikkawa, I.; Yorifuji, T. Synthesis 1980, 877.

[20] Yoshioka, M.; Tsuji, T.; Shoichiro, U. Tetrahedron Lett. 1980, 21, 351.

[21] Yanagisawa, H.; Ando, A. Tetrahedron Lett. 1982, 23, 3379.

[22] Cooper, R. D. G.; Kinnick, M. D.; Peters, L. R.; Morin, Jr. Synth. Commun. 1988, 18, 763.

[23] Chmielewski, M.; Kaluz, A.; Grodner, J.; Urbanski, R. ACS Symp. Ser. 1992, 494, 50.

[24] Chmielewsk, M.; Kaluz, A.; Furman, B. Chem. Commun. 1996, 2689.

[25] Kaluza, Z.; Park, H. Synlett 1996, 895.

[26] Kaluza, Z.; Lysek, R. Tetrahedron: Asymmetry 1997, 8, 2553.

[27] Kaluza, Z.; Lysek, R. Tetrahedron Lett. 1998, 39, 8349.

[28] Kaluza, Z. Tetrahedron Lett. 1999, 40, 1025.

[29] Kaluza, Z.; Furman, B.; Krajewski, P.; Chmielewski, M. Tetrahedron 2000, 56, 5553.

[30] Kaluza, Z.; Kazimierski, A.; Lewandowski, K.; Suwinska, K.; Szczesna, B.; Chmielewski, M. Tetrahedron 2003, 59, 5893.

[31] Koziol, A.; Frelek, J.; Woznica, M.; Furman, B.; Chemielewski Eur. J. Org. Chem. 2009, 338.

[32] Yamada, H.; Kawate, T.; Matsumizu, M.; Nishda, A.; Yamaguchi, K.; Nagakawa, M. J. Org. Chem. 1998, 63, 6348.

[33] Wanner, M. J.; Hiemstra, H. Angew. Chem., Int. Ed. 2007, 46, 7485.

[34] Raheem, I. T.; Thiara, P. S.; Peterson, E. A.; Jacobsen, E. N. J. Am. Chem. Soc. 2007, 129, 13404.

[35] Raheem, I. T.; Thiara, P. S.; Jacosen, E. N. Org. Lett. 2008, 10, 1577.

[36] Uraguchi, D.; Terada, M. J. Am. Chem. Soc. 2004, 126, 5356.

[37] Akiyama, T.; Saitoh, Y.; Morita, K.; Fuchibe, K. Adv. Synth. Catal. 2005, 347, 1523.

[38] Rueping, M.; Sugiono, E.; Azap, C.; Theissmann; Bolte, M. Org. Lett. 2005, 7, 3781 .

[39] Borsuk, K.; Suwinska, K.; Chmielewski, M. Tetrahedron: Asymmetry 2001, 12, 979.

[40] Borsuk, K.; Kazimierski, A.; Solecka, J.; Urbanczyk-Lipkowska, Z.; Chmielewski, M. Carbohydr. Res. 2002, 337, 2005.

[41] Fruchtel, J.; Jung, G. Angew. Chem., Int. Ed. Engl. 1996, 35, 17.

[42] Thompson, L. A.; Ellman, J. A. Chem. Rev. 1996, 96, 555.

[43] Hermkens, P. H. H.; Ottenheijm, H. C. J.; Rees, D. C. Tetrahedron 1996, 52, 4527.

[44] Hermkens, P. H. H.; Ottenheijm, H. C. J.; Rees, D. C. Tetrahedron 1997, 53, 5643 .

[45] Booth, S.; Hermkens, P. H. H.; Ottenheijm, H. C. J.; Rees, D. C. Tetrahedron 1998, 54, 15385.

[46] Ruhland, B.; Bhandari, A.; Gordon, E. M.; Gallop, M. A. J. Am. Chem. Soc. 1996, 118, 253.
[47] Ruhland, B.; Bombrun, A.; Gallop, M. A. J. Org. Chem. 1997, 62, 7820.

[48] Pei, Y.; Houghten.; Kiely, J. S. Tetrahedron Lett. 1997, 38, 3349.

[49] Benaglia, M.; Cinquini, M.; Cozzi, F. Tetrahedron Lett. 1999, 40, 2019.

[50] Furman, B.; Thurmer, R.; Kaluza, Z. Angew. Chem., Int. Ed. 1999, $38,1121$.

[51] De Witt. S. H, Kiely, J. S.; Stankovic, C. J.; Schroeder, M. C.; Reynolds Cody, D. M.; Pavia, M. R. Proc. Natl. Acad. Sci. U. S. A. 1993, 90, 6909.

[52] Dressman, B. A.; Spangle, L. A.; Kaldor, S. W. Tetrahedron Lett. 1996, 37, 937.

[53] Kolodziej, S. A.; Hamper, B. C. Tetrahedron Lett. 1996, 37, 5277.

[54] Tietze, L. F.; Steinmetz, A.; Balkenhohl, F. Bioorg. Med. Chem. Lett. 1997, 7, 1303.

[55] Matthews, J.; Rivero, R. A. J. Org. Chem. 1997, 62, 6090.

[56] Kim, S. W.; Ahn, S. Y.; Koh, J. S.; Lee, J. H.; Ro, S.; Cho, H. Y. Tetrahedron Lett. 1997, 38, 4603.

[57] Fantauzzi, P. P.; Yager, K. M. Tetrahedron Lett. 1998, 39, 1291.

[58] Stadlwieser, J.; Ellmerer-Muller, E. P.; Tako, A.; Maslouh, N.; Bannwarth, W. Angew. Chem., Int. Ed. 1998, 37, 1402.

[59] Van Maarseveen, J. H.; Den Hartog, J. A. J.; Engelen. V.; Finner, E, Tetrahedron Lett. 1996, 37, 8249.

[60] Veerman, J. J. N.; Van Maarseveen, J. H.; Visser, G. M.; Kruse, C. G.; Schoemaker, H. E.; Hiemstra, H.; Rutjes, F. P. J. T. Eur. J. Org. Chem. 1998, 2583.

[61] Nicolaou, K. C.; Winssinger, N.; Pastor, J.; Ninkovic, S.; Sarabia, F.; He, Y.; Vourloumis, D.; Yang, Z.; Li, T.; Giannakakou, P.; Hamel, E. Nature 1997, 387, 268.

[62] Nicolaou, K. C.; Pastor, J.; Winssinger, N.; Murphy, F. J. J. Am. Chem. Soc. 1998, 120, 5132.

[63] Hanessian, S.; Huynh, H. K. Synlett 1999, 102.

[64] Wendeborn, S.; De Mesmaeker, A.; Brill, W. K. D. Synlett 1998, 865.

[65] Furman, B.; Thurmer, R.; Kaluza, Z.; Voelter, W.; Chmielewski, M. Tetrahedron Lett. 1999, 40, 5909.

[66] Hauser, F. M.; Ellenberger, S. R. Synthesis 1987, 324.

[67] Alcaide, B.; Aly, M. F.; Sierra, M. A. Tetrahedron Lett. 1995, 36, 3401.

[68] Alcaide, B.; Aly, M. F.; Sierra, M. A. J. Org. Chem. 1996, 61, 8819.

[69] Kaluza, Z.; Lysek, R. Tetrahedron: Asymmetry 1997, 15, 2553.

[70] Baldwin, J. E. J. C. S. Chem. Commun. 1976, 734.

[71] France, S.; Guerin, D. J.; Miller, S. J.; Lectka, T. Chem. Rev. 2003, 103, 2985.

[72] Chen, Y.; McDaid, P.; Deng, L. Chem. Rev. 2003, 103, 2965.

[73] Pracejus; Forschr, H. Chem. Forsch. 1967, 8, 493.

[74] Kacprzak, K.; Gawronski, J. Synthesis 2001, 961.

[75] Tian, S. K.; Chen, Y.; Hang, J.; Tang, L.; McDaid, P.; Deng, L. Acc. Chem. Res. 2004, 37, 621.

[76] Palomo, C.; Oiarbide, M.; Lopez, R. Chem. Soc. Rev. 2009, 38, 632.

[77] Koziol, A.; Furman, B.; Frelek, J.; Woznica, M.; Altieri, E.; Chmielewski, M. J. Org. Chem. 2009, 74, 5687.

[78] Koziol, A.; Altieri, E.; Furman, B.; Solecka, J.; Chmielewski, M. ARKIVOC 2011, 37.

[79] Cambell, M. M.; Nelson, K. H.; Cameron, A. F. J. Chem. Soc., Chem. Commun. 1979, 532.

[80] Arnoldi, A.; Merlini, L.; Scaglioni, L. J. Heterocycl. Chem. 1987, 75.

[81] Buynak, J. D.; Mathew, J.; Rao, M. N.; Haley, E.; George, C.; Siriwardane, U. J. Chem. Soc., Chem Commun. 1986, 941.

[82] Bagrat, A. S.; Ljudmila, L. T. Chem. Rev. 2013, 113, 699. 
[83] Vassilev, L. T.; Vu, B. T.; Graves, B.; Carvajal, D.; Podlaski, F.; Filipovic, Z.; Kong, N.; Kammlott, U.; Lucacs, C.; Klein, C.; Fotouhi, N.; Liu, E. A. Science 2004, 303, 844.

[84] Grosse, L.; Paquet, S.; Caron, P.; Fazli, L.; Rennie, P. S.; Belanger, A.; Barbier, O. Cancer Res. 2013, 73, 6963.

[85] Tabolin, A. A.; Ioffe, S. L. Chem. Rev. 2014, 114, 5426

[86] Carbone, A.; Pennati, M.; Parrino, B.; Lopergolo, A.; Barraja, P.; Montalbano, A.; Spano, V.; Sbarra, S.; Doldi, V.; Cesare, M. D.; Cirrincione, G.; Diana, P.; Zaffaroni, N. J. Med. Chem. 2013, 56, 7060 .

[87] Buynak, J. D.; Rao, M. N.; Pajouhesh, H.; Chandrasekaran, R. Y.; Finn, K.; Meester, P.; Chu, S. C. J. Org. Chem. 1985, 50, 4245.

[88] Buynak, J. D.; Pajouhesh, H.; Lively, D. L.; Ramalakshimi, Y. J. Chem. Soc., Chem. Commun. 1984, 948.

[89] Moriconi, E. J.; Kelly, J. F. J. Org. Chem. 1968, 33, 3036.

[90] Buynak, J. D.; Mathew, J.; Narayana, R. M. J. Chem. Soc., Chem. Commun. 1986, 941.

[91] Buynak, J. D.; Rao, M. N.; Chandrasekran, R. Y.; Haley, E. Tetrahedron Lett. 1985, 26, 5001.

[92] Kaluza, Z.; Furman, B.; Patel, M.; Chmielewski, M. Tetrahedron: Asymmetry 1994, 5, 2179.

[93] Lo, Y. S.; Sheehan, J. C. J. Am. Chem. Soc. 1972, 94, 8253.

[94] Buckley, T. E.; Rapoport. H. J. Am. Chem. Soc, 1982, 104, 4446.

[95] Lysek, R.; Zofia, U.-L.; Chmielewski, M. Tetrahedron 2001, 57, 1301.

[96] Lysek, R.; Furman, B.; Kaluza, Z.; Frelek, J.; Suwinska, K.; Chmielewski, M. Tetrahedron: Asymmetry 2000, 11, 3131.

[97] Lysek, R.; Grzeszczyk, B.; Furman, B.; Chmielewski, M. Eur. J. Org. Chem. 2004, 4177.

[98] Lysek, R.; Kaluza, Z.; Furman, B.; Chmielewski, M. Tetrahedron 1998, 54, 14065.

[99] Cierpucha, M.; Solecka, J.; Frelek, J.; Szczukiewicz, M.; Chmielewski, M. Biorg. Med. Chem. 2004, 12, 405.

[100] Lee, M.-R.; Stahl, S. S.; Gellman, S. H. Org. Lett. 2014, 16, 3848.

[101] Buynak, J. D.; Mathew, J.; Rao, M. N.; Haley, E.; George, C.; Siriwardane, U. J. Chem. Soc., Chem. Commun. 1987, 735.

[102] Hui, X.-P.; Wang, Q.; Wang, F.; Zhang, Z. Y.; Guan, Z. W. Chin. J. Org. Chem. 2006, 26, 1704 (in Chinese). (惠新平, 王勤, 王芳, 张自义, 管作武, 有机化学, 2006, 26, 1704.)

[103] Kawamura, Y.; Yasuda, Y.; Mayama, M.; Tanaka, K. J. Antibiot. 1982, 35, 10.

[104] Shoji, J.; Hinoo, K.; Sakazaki, R.; Tsuji, N.; Nagashima, K.; Matsumoto, K.; Takahashi, Y.; Kozuki, S.; Hattori, T.; Kondo, E.; Tanaka, K. J. Antibiot. 1982, 35, 15.

[105] Tsuji, N.; Nagashima, K.; Kobayashi, M.; Shoji, J.; Kato, T.; Terui, Y.; Nakai, H.; Shiro, M. J. Antibiot. 1982, 35, 24.

[106] Murakami, K.; Doi, M.; Yoshida, T. J. Antibiot. 1982, 35, 39

[107] Arisawa, M.; Then, R. L. J. Antibiot. 1982, 35, 1578.

[108] Brenner, D. G. J. Org. Chem. 1985, 50, 18.

[109] Buynak, J. D.; Wu, K.; Bachmann, B.; Khasnis, D.; Hua, L.; Nguyen, H. K.; Carver, C. C. J. Med. Chem. 1995, 38, 1022.

[110] Danh, T. T.; Bocian, W.; Kozerski, L.; Szczukiewicz, P.; Frelek, J.; Chemielewski, M. Eur. J. Org. Chem, 2005, 429.

[111] Smith, S. R.; Douglas, J.; Prevtet, H.; Shapland, P.; Smith, A. D. J. Org. Chem. 2014, 79, 1626.

[112] Palomo, C.; Aizpurua, J. M.; Ganboa, L.; Oiarbide, M. Curr. Med. Chem. 2004, 11, 1837.

[113] Fu, N.; Tidwell, T. T. Tetrahedron 2008, 64, 10465.

[114] Staudinger, H. Liebigs Ann. 1907, 356, 51.

[115] Tarui, A.; Kawashima, N.; Kawakita, T.; Sato, K.; Omote, M.; Ando, A. J. Org. Chem. 2013, 78, 7903.
[116] Ye, J.-W.; Yang, C.; Yang, D. B.; Liu, F. M. Chin. J. Org. Chem. 2009, 29, 414 (in Chinese).

(叶嘉炜，杨春，杨德保，刘方明，有机化学, 2009, 29, 414.)

[117] Kim, I.; Roh, S. W.; Lee, D. G.; Lee, C. Org. Lett. 2014, 16, 2482.

[118] Zhang, H. M.; Gao, Z. H.; Ye, S. Org. Lett. 2014, 16, 3079.

[119] Casarrubios, L.; Larramona, C.; Olivan, M.; Onate, E. Organometallics 2014, 33, 1820

[120] McGuire, M. A.; Hegedus, L. S. J. Am. Chem. Soc. 1982, 104, 5538.

[121] Lei, J.; Yong, L.; Wu, C. Z.; Huang, X.; Xu, J. X. Chem. Res. Chin. Univ. 2005, 21, 59.

[122] Qi, H. Z.; Yang, Z. H.; Xu, J. X. Synthesis 2011, 5, 723.

[123] Ongania, K. H.; Schwarzenbrunner, U.; Humer, K. Monatshefte fur Chemie 1984, 115, 215.

[124] Hegedus, L. S.; McGuire, M. A.; Schultze, L. M.; Yijun, C.; Anderson, O. P. J. Chem. Soc. 1984, 106, 2680.

[125] Hegedus, L. S. Acc. Chem. Res. 1995, 28, 299.

[126] Reed, A. D.; Hegedus, L. S. J. Org. Chem. 1995, 60, 3787.

[127] Merino, I.; Hegedus, L. S. Organmetallics 1995, 14, 2522.

[128] Kobbing, S.; Mattay, J.; Raabe, G. Chem. Ber. 1993, 126, 1849.

[129] Wulff, W. D.; Anderson, B. A.; Toole, A. J.; Xu, Y.-C. Inorg. Chim. Acta 1994, 220, 215

[130] Lin, D.-Z.; Wang, J.; Liu, H. Chin. J. Org. Chem. 2013, 33, 2098 (in Chinese).

(林岱宗, 王江, 柳红, 有机化学, 2013, 33, 2098.)

[131] O'Sullivan, D.; Mclaughlin, R. P.; Clemitshaw, K. C.; Sodeau, J. R. J. Phys. Chem. A 2014, 118, 9890.

[132] Buss, J. A.; Edouard, G. A.; Cheng, C.; Shi, J.; Agapie, T. J. Am. Chem. Soc. 2014, 136, 11272.

[133] Bagchi, V.; Paraskevopoulou, P.; Das, P.; Chi, L. G.; Wang, Q. W.; Choudhury, A.; Cronin, L.; Mitrikas, G.; Sanakis, Y.; Stavropoulos, P. J. Am. Chem. Soc. 2014, 136, 11362.

[134] Hegedus, L. S.; Weck, G. D.; D'Andrea, S. J. Am. Chem. Soc. 1988 110.

[135] Borel, C.; Hegedus, L. S.; Krebs, J.; Satoh, Y. J. Am. Chem. Soc. 1987, 109.

[136] Colson, P.-J.; Hegedus, L. S. J. Org. Chem. 1993, 58.

[137] Mori, M.; Kanda, N.; Ban, Y. J. Chem. Soc., Chem. Commun. 1986.

[138] Brackmann, F.; Meijere, D. A. Chem. Rev. 2007, 107, 4493.

[139] Newman, S. G.; Lautens, M. J. Am. Chem. Soc. 2011, 133, 1778.

[140] Trost, B. M.; Jonasson, C.; Wuchrer, M. J. Am. Chem. Soc. 2001, $123,12736$.

[141] Trost, B. M.; Jonasson, C. Angew. Chem. 2003, 115, 2109.

[142] Yu, C.-M.; Kim, Y.-M.; Kim, J.-M. Synlett 2003, 1518.

[143] Norret, M.; Sherburn, M. S. Angew. Chem., Int. Ed. 2001, 40, 4074.

[144] Nicolau, K. C.; Li, J. Angew. Chem., Int. Ed. 2001, 40, 4264.

[145] Neumann, H.; J, A.; Wangelin, V.; Gordes, D.; Spannenberg, A.; Beller, M. J. Am. Chem. Soc. 2001, 123, 8398.

[146] Alcaide, B.; Almendros, P.; Aragoncillo, C. Chem. Eur. J. 2002, 8 , 1719.

[147] Alcaide, B.; Almendros, P.; Aragoncillo, C.; Rodriguezacebes, R. J. Org. Chem. 2001, 66, 5208.

[148] Alcaide, B.; Almendros, P.; Aragoncillo, C. Org. Lett. 2000, 2, 1411.

[149] Alcaide, B.; Almendros, P. Chem. Soc. Rev. 2001, 30, 226.

[150] Alcaide, B.; Almendros, P. Synlett 2002, 381.

[151] Alcaide, B.; Almendros, P.; Aragoncillo, C.; Redondo, M. C. Eur. J. Org. Chem. 2005, 98. 